\title{
Model and Characteristic Simulation Analysis of a Two-Speed Transmission System
}

\author{
Zhigang Chen, Feng Xie and Zhihui Liu \\ School of Mechanical and Energy Engineering, Shaoyang University, Shaoyang 422000, China. \\ Key Laboratory of Hunan Province for Intelligent Manufacturing of Efficient Power System, Shaoyang University, \\ Shaoyang 422000, China.
}

\author{
Hongzhi Yan \\ School of Mechanical and Electrical Engineering, Central South University, Changsha 410083, China. \\ The State Key Laboratory of High-performance and Complex Manufacturing, Central South University, Changsha \\ 410083, China. E-mail:154135060@qq.com.
}

\begin{abstract}
(Received 29 January 2021; accepted 3 May 2021)
One of the main tasks in the research of a helicopter two-speed transmission system was to improve its dynamic characteristics. For the low gear mode of the system, a dynamic model was established by using the lumped parameter method, the method of Runge-Kutta was used to solve the nonlinear dynamic system equations. The effect of the gear module on the dynamic transmission error, dynamic load of the gear pair and the dynamic windup angle of a one-way clutch were studied. And the effect of the one-way clutch torsional stiffness on the dynamic transmission error and dynamic load of the gear pair was also studied. The results show that: 1) the dynamic transmission error of the gear pair decreases and the dynamic load of the gear pair increases with the increase of the gear module at the lower range of excitation frequencies; 2) the dynamic windup angle of the one-way clutch increases with an increase of the gear module. 3) the dynamic transmission error of the gear pair and the maximum dynamic load increases with an increase of the one-way clutch torsional stiffness at the lower and medium range of excitation frequencies. The above results can provide reference for the subsequent upgrade and improvement of the two-speed transmission system.
\end{abstract}

\section{INTRODUCTION}

Helicopters have superior characteristics, including vertical take-off and landing, hovering in the air, high mobility, and no need for airport runways. With the development of the global economy and defense technology, the demand for helicopters has become more prominent. In order to improve the mobility and fuel economy of helicopters, a two-speed transmission system has been introduced in the helicopter's transmission system. Figure 1 shows the principle of the system structure. It is observed that the coupling system consists of a one-way clutch, friction clutch, and a spur gear pair. By controlling the engagement and disengagement of the friction clutch, the one-way clutch automatically disengages and engages, respectively, to realize the two-speed transmission speed output. When the friction clutch 6 is engaged, the output end speed of the oneway clutch 3 is higher than the input end speed. Therefore, the clutch automatically turns to the one-way state, which results in a high gear corresponding to the hovering state of the helicopter. On the other hand, when friction clutch 6 is released, the one-way clutch 3 undergoes an automatic engagement and the low-gear mode is achieved. The essence of this mode is a coupling system composed of a two-stage gear transmission and a one-way clutch, which corresponds to the helicopter cruise state. The present study is based on the coupling system of this state.

Currently, the one-way clutch and gear transmission system

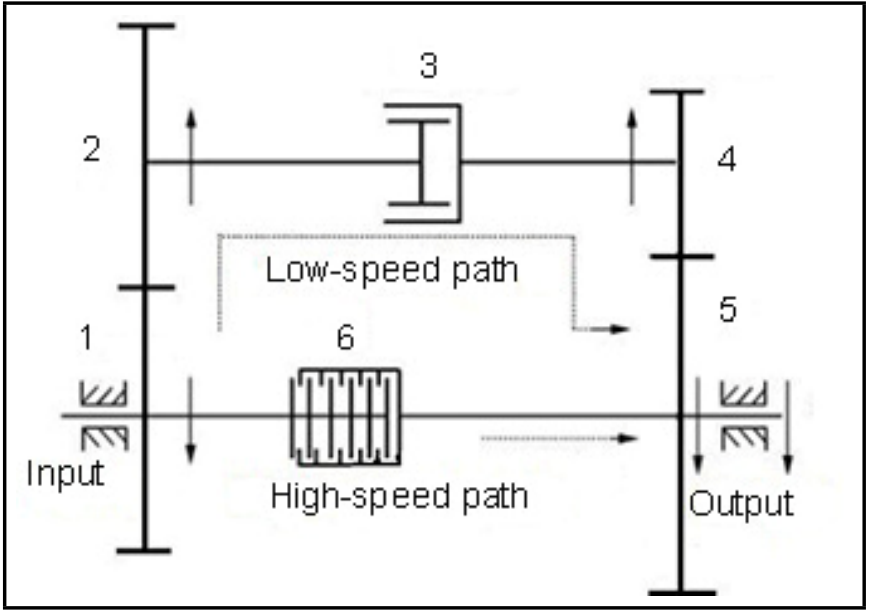

Figure 1. Schematic configuration of the helicopter two-speed transmission system. 1 - Low speed input driving gear. 2 - Low speed input driven gear. 3 One-way clutch. 4 - Low speed output end driving gear. 5 - Low speed output driven gear. 6 - Friction clutch.

are widely used in conventional helicopters and they have been the subject of several research papers.

Considering the pure gear system, several in-depth studies were carried out mainly from the aspects of system modeling, dynamic equation solving, the dynamic characteristic analysis, and dynamic optimization design. The system modeling mainly adopts methods such as the lumped parameter method, finite element method, bond graph method, and test method. 
Tuplin $^{1}$ proposed the gear transmission system as an elastic mechanical vibration model. Moreover, the lumped parameter method was used to establish the mechanical model of the gear transmission system to provide a foundation for the research and analysis of the dynamics of the gear transmission system. A certain theoretical basis gradually changes the analysis and research of the gear dynamic load from shock theory to vibration theory. Sainte-Marie et al., ${ }^{2}$ applied the finite element method (FEM) to investigate the correlation between the dynamic transmission error and dynamic meshing load of spur gears and helical gears, and then verified the obtained correlations through experiments. Moreover, Gradu et al., ${ }^{3}$ applied the finite element model to obtain the dynamic response of the system with different planetary wheel spacing. The dynamic equations are generally solved by numerical methods and harmonic balance methods. The commonly used numerical integration methods include the central difference method, the Wilson- $\theta$ method, the Newmark- $\beta$ method, and the Runge-Kutta method. Kahraman and Singh ${ }^{4}$ established a single-degree-of-freedom nonlinear gear model with clearance and used numerical methods and harmonic balance methods to simulate the system and understand its transmission characteristics. Chaari et al., ${ }^{5}$ used an iterative method to calculate the dynamic response of the planetary gear transmission system. Moreover, they studied the influence of the timevarying meshing stiffness, eccentricity error, and tooth profile modification on the dynamic response. Al-shyyab ${ }^{6}$ applied the harmonic balance method to investigate the system response and compared the results with that of the numerical integration method.

The dynamic characteristics of the gear system include inherent characteristics, parameter stability, and dynamic response. ${ }^{7}$ Moreover, inherent characteristics include natural frequency and mode shape, which are the basic problems of gear dynamics research. Therefore, these characteristics are of great significance in the dynamic response of the system, the generation and transmission of dynamic loads, and the vibration behavior of the system. ${ }^{8,9}$ By studying the parameter stability of the gear transmission, the stable area of the system operation can be determined, thus avoiding the instability of the transmission system, and providing a basis for the system design and vibration control. Sun Zhi-min ${ }^{10}$ used numerical integration to obtain the simple harmonic, non-harmonic singleperiod, sub-harmonic, quasi-periodic, and chaotic steady-state forced response under different parameter conditions. The main purpose of investigating the dynamic response of the gear transmission system is to reduce the transmission of the dynamic excitation and the vibration of each component in the system, thereby increasing the service life and reducing system noise and vibration. Non-linear factors that affect the dynamic response of gears include: gear time-varying meshing stiffness, tooth side clearance, bearing clearance and tooth surface friction. Nakamura ${ }^{11}$ studied gears with backlash. Moreover, Liu et al., ${ }^{12}$ analyzed the influence of bearing clearance and tooth side clearance on the gear meshing force. Wang et al., ${ }^{13}$ investigated the time-varying clearance caused by the gear eccentricity and load changes and analyzed the influence of load and gear eccentricity on the dynamic transmission error of the sys- tem. Liu et al., ${ }^{14}$ analyzed the effect of the gear meshing force on the tooth surface wear and studied the effect of the tooth surface wear on the dynamic transmission error of gear pairs.

Dynamic investigations of the pure one-way clutch system mainly focus on the mechanical characteristics of the clutch engagement process and the influence of the working type of the diagonal brace on the dynamic characteristics. $\mathrm{Xu}$ and Lowen ${ }^{15}$ established the nonlinear numerical analysis model of the brace clutch and obtained the mathematical expressions of the contact force and slip angle. Based on the theory of contact mechanics, Chassapis and Lowen ${ }^{16}$ established a dynamic model for analyzing the nonlinear stiffness of the clutch during the engagement process and the transmission state. Centea and Rahnejat ${ }^{17}$ investigated the influence of the clutch's geometrical size, inertia and the contact force between the wedge and the raceway. Moreover, they established a nonlinear multibody dynamics model of the clutch and analyzed the torsional vibration of the clutch. Yan Hong-zhi and Yang Bing ${ }^{18,19}$ studied the engagement performance of the PCE clutch during wedging and its contact characteristics under different working conditions. Moreover, they used the Marc method to establish a mathematical model and perform a numerical simulation. Accordingly, they obtained the wedge and clutch of the PCE clutch in the transmission state, the contact stress of the raceway, and the simulation of the distribution law of the contact stress along the axial direction of the diagonal brace. $\mathrm{Wu}$ Kai et al. ${ }^{20,21}$ designed the diagonal brace clutch with three different profile wedges, including the Archimedes profile, a logarithmic curve and a circular arc, and established a dynamic simulation analysis model. Then, the diagonal brace block profile was proposed accordingly. It was found that the clutch with a logarithmic profile had a better working performance in comparison with the two other profiles. Niranjan Hiremath and D.Malikarjuna Reddy ${ }^{22,23}$ through comparative analysis, reveal that acoustic technology can detect bearing failure more effectively than vibration technology and further investigated the effect for system friction and vibration of $\mathrm{TiN}$ and $\mathrm{AlCrN}$ based PVD coatings.

An integrated system is composed of a one-way clutch and a pair of spur gear transmission pairs. It should be indicated that this system is more complex than a simple clutch or gear system. Considering the dynamic behavior of this system, the coupling nonlinearity between the one-way clutch and the characteristics of the gear transmission pair was studied. Investigation of the nonlinear dynamic characteristics of such systems can theoretically explain the nonlinear dynamic behaviors of systems, and obtain general dynamic design methods for them. Meanwhile, it can also be applied to theoretically analyze the nonlinear dynamic characteristics of complex integrated systems. Although this type of system is widely used in diverse applications, there are few studies of the nonlinear characteristics of the one-way clutch-single pair gear transmission systems. Gill-Jeong Cheon ${ }^{24}$ studied the integration of a one-way clutch and spur gear transmission pairs with nonlinear dynamic characteristics. First, a three degrees-of-freedom torsional dynamic model of the system was established, and numerical integration was used to simulate and solve the problem. The study found that compared to a pure gear transmis- 


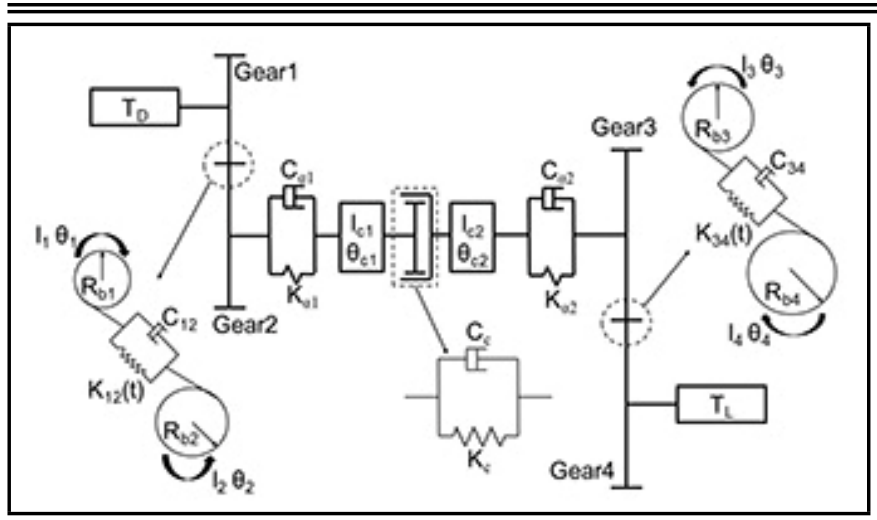

Figure 2. Sketch of the two-stage gear transmission system model with oneway clutch.

sion system, the one-way clutch can effectively reduce torsional vibration. Moreover, it was found that the system obtained a typical non-linear behavior such as jumping characteristics. However, the system modeling did not consider the influence of the lateral vibration of the one-way clutch system and the coupling influence of nonlinear factors such as bearing contact stiffness, damping, and clearance. Based on the model provided by Gill-Jeong Cheon, Wang Peng ${ }^{25}$ further considered the influence of the gear transmission pair tooth frequency error. Using the variable step length Gill numerical algorithm, the bifurcation characteristics of the system with the excitation frequency and the path to chaos were used. The path and parameter influence law was discussed. Mallikarjuna Reddy, et al., ${ }^{26}$ verified the accuracy of the mathematical model of vehicle ride comfort by MATLAB.

The abovementioned analysis shows that although some research has been established on the one-way clutch, gear transmission system and the coupling system combining the two in the early stage, the influence of the intermediate connecting shaft and gear module parameters on the system has yet to be studied. Therefore, in the present study, a five-degreesof-freedom nonlinear dynamic model, including gear meshing stiffness, meshing damping, clutch torsional stiffness, torsional damping, intermediate connecting shaft torsional stiffness, torsional damping, and gear static transmission error was proposed. Moreover, the Runge-Kutta method was used to solve this nonlinear system. The research results provide references for the dynamic design and control of such transmission systems for technicians and engineering designers.

\section{MATHEMATICAL MODEL}

Figure 2 illustrates the two-stage gear transmission system with a one-way clutch. It is observed that the system is composed of gear 1, gear 2, one-way clutch, gear 3, and gear 4 in a series. Gear 1 meshes with gear 2 to form a gear pair. Moreover, gear 3 meshes with gear 4 to form a gear pair.

It was assumed that the gears of the system are all involute spur gears without modification. Therefore, the torsion and bending deformation of the input shaft and output shaft were ignored. Moreover, according to the schematics of the system model, the lumped parameter method and Newton's law were used. The motion differential equation of the system was mathematically expressed as the following:

$$
\left\{\begin{array}{l}
I_{1} \ddot{\theta}_{1}+C_{12}\left(R_{b 1} \dot{\theta}_{1}-R_{b 2} \dot{\theta}_{2}-\dot{e}_{12}(t)\right) R_{b 1} \\
+k_{12}(t) f\left(R_{b 1} \theta_{1}-R_{b 2} \theta_{2}-e_{12}(t)\right) R_{b 1}-T_{D}=0 \\
\ddot{I}_{2} \ddot{\theta}_{2}+C_{12}\left(R_{b 1} \dot{\theta}_{1}-R_{b 2} \dot{\theta}_{2}-\dot{e}_{12}(t)\right) R_{b 2} \\
+k_{12}(t) f\left(R_{b 1} \theta_{1}-R_{b 2} \theta_{2}-e_{12}(t)\right) R_{b 2} \\
+C_{\alpha 1}\left(\dot{\theta}_{2}-\dot{\theta}_{1}\right)+k_{\alpha 1}\left(\theta_{2}-\theta_{c 1}\right)=0 \\
I_{c 1} \ddot{\theta}_{c 1}-C_{\alpha 1}\left(\dot{\theta}_{2}-\dot{\theta}_{1}\right)-k_{\alpha 1}\left(\theta_{2}-\theta_{c 1}\right)+T_{C}=0 \\
I_{c 2} \ddot{\theta}_{c 2}+C_{\alpha 2}\left(\dot{\theta}_{c 2}-\dot{\theta}_{3}\right)+k_{\alpha 2}\left(\theta_{c 2}-\theta_{3}\right)-T_{C}=0 \\
I_{3} \ddot{\theta}_{3}+C_{34}\left(R_{b 3} \dot{\theta}_{3}-R_{b 4} \dot{\theta}_{4}-\dot{e}_{34}(t)\right) R_{b 3} \\
+k_{34}(t) f\left(R_{b 3} \theta_{3}-R_{b 4} \theta_{4}-e_{34}(t)\right) R_{b 3} \\
-C_{\alpha 2}\left(\dot{\theta}_{2}-\dot{\theta}_{3}\right)-k_{\alpha 2}\left(\theta_{c 2}-\theta_{3}\right)=0 \\
\ddot{I}_{4}+C_{34}\left(R_{b 3} \dot{\theta}_{3}-R_{b 4} \dot{\theta}_{4}-\dot{e}_{34}(t)\right) R_{b 4} \\
+k_{34}(t) f\left(R_{b 3} \theta_{3}-R_{b 4} \theta_{4}-e_{34}(t)\right) R_{b 4}+T_{L}=0
\end{array}\right.
$$

where $e_{12(34)}(t)$ represents the comprehensive tooth frequency error of the meshing gears, and it uses the meshing tooth frequency $\omega_{i}(i=1,2)$ as the periodic function of the changing frequency: $e_{12(34)}(t)=e_{a i} \cos \left(\omega_{i} t+\phi_{h i}\right)(i=1,2)$. Moreover, $T_{D}, T_{L}$, and $T_{C}$ denote the driving torque acting on gear 1 , the load torque output by gear 4 and the torque of the one-way clutch, respectively. Furthermore, $f\left(x_{i}\right)=$ $\left\{\begin{array}{l}x_{i}-b_{i}, x_{i}>b_{i} \\ 0, x_{i} \leq b_{i} \\ x_{i}+b_{i}, x_{i}<b_{i}\end{array} \quad, C_{12(34)}=2 \xi_{1(2)} \sqrt{\frac{k_{0}}{\frac{r_{b 1(3)}^{2}}{I_{1(3)}(3)} I_{2(4)}^{2}}}\right.$ represent the nonlinear function of the meshing force of the gear pair with backlash and the damping coefficient of the gears meshing respectively. $\zeta_{1(2)}$ represent the gear pair with the meshing damping ratio of the gear pair.

$R_{b i}, I_{i}$, and $\theta_{i}(i=1.2 .3 .4)$ represents the base circle radius, the moment of inertia and torsional displacement of gears 1,2 , 3 , and 4 , respectively.

$I_{c i}$ and $\theta_{c i}(i=1.2)$ represents the moment of inertia and torsional displacement of the primary and driven ends of the one-way clutch, respectively.

$C_{\alpha i}$ and $k_{\alpha i}(i=1.2)$ represent the damping coefficient and the torsional stiffness of the intermediate connecting shaft, respectively.

$K_{c}$ and $C_{c}$ denote the torsional stiffness of the overrunning clutch and the torsional damping of the overrunning the clutch, respectively. Moreover, $b_{i}(i=1,2)$ represents half of the backlash of the two pairs of meshing gears.

$k_{12(34)}(t)$ represents the time-varying stiffness of the gear pairs, refer to the studies of, ${ }^{1,25}$ expressed in Fourier series: $k_{12(34)}((t))=k_{01(2)}+\sum_{i=1}^{n} k_{h i} \cos \left(i \omega_{1(2)} t-\phi_{i}\right)$, $k_{01(2)} / k_{t p}=\varepsilon, k_{h i} / k_{t p}=\sqrt{2-2 \cos (2 \pi i(\varepsilon-1))} / \pi i,{ }_{i}=$ $\alpha \tan ((1-\cos (2 \pi i(\varepsilon-1))) /(\sin (2 \pi i(\varepsilon-1))))$.

Where $k_{01(2)}$ and $k_{h i}$ represent the average meshing stiffness of the gears and the amplitude of the $i$-th harmonic, re- 
spectively. Moreover, $\phi_{i}$ and $\varepsilon$ are the phase angle of the $i$-th harmonic and the coincidence degree of the gear, respectively. By the first five harmonics, a more proficient accuracy can be obtained. Therefore, $i=5 ; \omega_{1(2)}$ is the meshing tooth frequency of the two pairs of gears.

According to Newton's second law, refer to the studies of, ${ }^{15,25}$ a one-way clutch model is established as follows:

$$
T_{C}=K_{c} f_{c}\left(\theta_{c 1}-\theta_{c 2}\right)^{a}+C_{c} \dot{f}_{c}\left(\theta_{c 1}-\theta_{c 2}\right)^{b} f_{c}\left(\theta_{c 1}-\theta_{c 2}\right)^{c}
$$

where coefficients $a, b$, and $c$ are the parameters introduced to the piecewise nonlinear clutch model. $a=1, b=1$, and $c=$ 0 are commonly used in the piecewise linear clutch models. $K_{c}$ and $C_{c}$ represent the torsional stiffness and damping of the one-way clutch, respectively. $C_{c}=2 Z_{c} \sqrt{\left(K_{c} I_{c}\right)}, z_{c}$ represent the damping ratio of the one-way clutch. Moreover, $f_{c}(x)=$ $\left\{\begin{array}{l}x, \dot{x}>0 \\ 0, \dot{x}<0\end{array} \quad, f_{c}\right.$ represent the nonlinear function of the oneway clutch.

The displacements of gear 1 , gear 2 , the one-way clutch, gear 3 , and gear 4 are defined on the meshing line as $x_{1}, x_{2}$, $x_{c 1}, x_{c 2}, x_{3}$, and $x_{4}$, respectively. The abovementioned displacements are equal to $x_{1}=R_{b 1} \theta_{1}, x_{2}=R_{b 2} \theta_{2}, x_{c 1}=$ $R_{b 2} \theta_{c 1}, x_{c 2}=R_{b 3} \theta_{c 2}, x_{3}=R_{b 3} \theta_{3}$ and $x_{4}=R_{b 4} \theta_{4}$, respectively. Considering the transformation of the equation of motion, the transformation results are as follows:

$$
\left\{\begin{array}{l}
m_{1} \ddot{x}_{1}+c_{12}\left(\dot{x}_{1}-\dot{x}_{2}-\dot{e}_{12}(t)\right) \\
+k_{12}(t) f\left(x_{1}-x_{2}-e_{12}(t)\right)-F_{D}=0 \\
m_{2} \ddot{x}_{2}+c_{12}\left(\dot{x}_{1}-\dot{x}_{2}-\dot{e}_{12}(t)\right) \\
+k_{12}(t) f\left(x_{1}-x_{2}-e_{12}(t)\right) \\
+\frac{c_{\alpha 1}}{R_{b 2}^{2}}\left(\dot{x}_{2}-\dot{x}_{c 1}\right)+\frac{k_{\alpha 1}}{R_{b 2}^{2}}\left(x_{2}-x_{c 1}\right)=0 \\
m_{c 1} \ddot{x}_{c 1}-\frac{c_{\alpha 1}}{R_{b 2}^{2}}\left(\dot{x}_{2}-\dot{x}_{c 1}\right)-\frac{k_{\alpha 1}}{R_{b 2}^{2}}\left(x_{2}-x_{c 1}\right) \\
+F_{c 1}=0 \\
m_{c 2} \ddot{x}_{c 2}+\frac{c_{\alpha 1}}{R_{b 3}^{2}}\left(\dot{x}_{c 2}-\dot{x}_{3}\right)-\frac{k_{\alpha 2}}{R_{b 3}^{2}}\left(x_{c 2}-x_{3}\right) \\
+F_{c 2}=0 \\
m_{3} \ddot{x}_{3}+c_{34}\left(\dot{x}_{3}-\dot{x}_{4}-\dot{e}_{34}(t)\right) \\
-k_{34}(t) f\left(x_{3}-x_{4}-e_{34}(t)\right) \\
-\frac{c_{\alpha 1}}{R_{b 3}^{2}}\left(\dot{x}_{c 2}-\dot{x}_{3}\right)-\frac{k_{\alpha 2}}{R_{b 3}^{2}}\left(x_{c 2}-x_{3}\right)=0 \\
m_{4} \ddot{x}_{4}+c_{34}\left(\dot{x}_{3}-\dot{x}_{4}-\dot{e}_{34}(t)\right) \\
+k_{34}(t) f\left(x_{3}-x_{4}-e_{34}(t)\right)+F_{L}=0
\end{array}\right.
$$

where, $m_{1}, m_{2}, m_{c 1}, m_{c 2}, m_{3}$, and $m_{4}$ represent the equivalent masses of gear 1 , gear 2 , one-way clutch active end, oneway clutch driven end, gear 3 and gear 4 on the meshing line, namely $m=I / R^{2}$, respectively. $F=T / R$ means equivalent force; $c_{12(34)}=C_{12(34)}$.

The meaning of the gear pair transmission error is the error between the actual position and the theoretical position of the driven wheel when the gear is meshing. The actual transmission error of gear transmission is composed of static transmission error $e_{12(34)}(t)$ and dynamic transmission error (DTE) $x_{i}-x_{i+1}=R_{b i} \theta_{i}-R_{b(i+1)} \theta_{(i+1)}$. The static transmission error is affected by factors such as manufacturing, installation, etc. The dynamic transmission error is affected by factors such as dynamic load, time-varying meshing stiffness, etc. The gear pair transmission error $q$ is mathematically expressed as follows:

$$
\left\{\begin{array}{l}
q_{1}=x_{1}-x_{2}-e_{12}(t) \\
q_{2}=x_{3}-x_{4}-e_{34}(t) \\
q_{3}=x_{2}-x_{c 1} \\
q_{4}=x_{c 2}-x_{3} \\
q_{c}=x_{c 1}-x_{c 2}
\end{array} ;\right.
$$

where $q_{1}, q_{2}, q_{3}, q_{4}$, and $q_{c}$ represent the relative correlations between gear 1 with gear 2 , gear 3 with gear 4 , gear 2 with a one-way clutch active end, one-way clutch driven end with gear 3, and one-way clutch active end with one-way clutch driven end displacement, respectively.

Performing an algebraic transformation with the Eq. (3) yields:

$$
\left\{\begin{array}{l}
\ddot{q}_{1}+\frac{c_{12} \dot{q}_{1}}{M_{1}}+\frac{k_{12}(t) f\left(q_{1}\right)}{M_{1}}-\frac{F_{D}}{m_{1}} \\
-\frac{c_{\alpha 1} \dot{q}_{3}}{m_{2} R_{b 2}^{2}}-\frac{k_{\alpha 1} q_{3}}{m_{2} R_{b 2}^{2}}=-\ddot{e}_{12}(t) \\
\ddot{q}_{2}+\frac{c_{34} \dot{q}_{3}}{M_{2}}+\frac{k_{34}(t) f\left(q_{2}\right)}{M_{2}}-\frac{F_{L}}{m_{4}} \\
-\frac{c_{\alpha 2} \dot{q}_{4}}{m_{3} R_{b 3}^{2}}-\frac{k_{\alpha 2} q_{4}}{m_{3} R_{b 3}^{2}}=-\ddot{e}_{34}(t) \\
\ddot{q}_{3}-\frac{c_{12} \dot{q}_{1}}{m_{2}}-\frac{k_{12}(t) f\left(q_{1}\right)}{m_{2}}-\frac{F_{c 1}}{m_{c 1}} \\
+\frac{c_{\alpha 1} \dot{q}_{3}}{M_{2 . c 1} R_{b 2}^{2}}+\frac{k_{\alpha 1} q_{3}}{M_{2 . c 1} R_{b 2}^{2}}=0 \\
\ddot{q}_{4}-\frac{c_{34} \dot{q}_{2}}{m_{3}}-\frac{k_{34}(t) f\left(q_{2}\right)}{m_{3}}-\frac{F_{c 2}}{m_{c 2}} \\
+\frac{c_{\alpha 2} \dot{q}_{4}}{M_{c 2.3} R_{b 3}^{2}}+\frac{k_{\alpha 2} q_{4}}{M_{c 2.3} R_{b 3}^{2}}=0 \\
\ddot{q}_{c}-\frac{c_{\alpha 1} \dot{q}_{3}}{m_{c 1} R_{b 2}^{2}}-\frac{k_{\alpha 1} q_{3}}{m_{c 1} R_{b 2}^{2}}-\frac{c_{\alpha 2} \dot{q}_{4}}{m_{c 2} R_{b 3}^{2}} \\
-\frac{k_{\alpha 2} q_{4}}{m_{c 2} R_{b 3}^{2}}+\frac{F_{c 1}}{m_{c 1}}+\frac{F_{c 2}}{m_{c 2}}=0
\end{array}\right.
$$

where $M_{1}=m_{1} m_{2} /\left(m_{1}+m_{2}\right)$ is the equivalent mass of the first pair of gear pairs; $M_{2}=m_{3} m_{4} /\left(m_{3}+m_{4}\right)$ is the equivalent mass of the second pair of gear pairs; $M_{2 . c 1}=$ $m_{2} m_{c 1} /\left(m_{2}+m_{c 1}\right)$ is the equivalent mass of gear 2 and the active end of the one-way clutch; $M_{c 2.3}=m_{c 2} m_{3} /\left(m_{c 2}+\right.$ $\left.m_{3}\right)$ is the equivalent mass of the driven end of the one-way clutch and gear 3; The dimensionless time scale $\tau=t \omega n$ and the displacement scale $b_{c}$ is described as follows:

$$
\left\{\begin{array}{l}
q_{1}=b_{c} \delta_{1} \\
q_{2}=b_{c} \delta_{2} \\
q_{3}=b_{c} \delta_{3} \\
q_{4}=b_{c} \delta_{4} \\
q_{c}=b_{c} \delta_{c}
\end{array}\right.
$$

Performing algebraic transformation with the Eq. (5), the fol- 
lowing equation is obtained:

$$
\left\{\begin{array}{l}
\ddot{\delta}_{1}+2 \frac{c_{12} \dot{\delta_{1}}}{2 M_{1} \omega_{n}}+\frac{k_{12}(\tau) f\left(\delta_{1}\right)}{M_{1} \omega_{n}^{2}}-\frac{F_{D}}{b_{c} m_{1} \omega_{n}^{2}} \\
-2 \frac{c_{\alpha_{1}} \dot{\delta}_{3}}{2 m_{2} R_{b 2}^{2} \omega_{n}}-\frac{k_{\alpha 1}}{\delta}{ }_{3} m_{2} R_{b 2}^{2} \omega_{n}^{2}=-\ddot{e}_{12}(\tau) \\
\ddot{\delta}_{2}+2 \frac{c_{34} \dot{\delta_{2}}}{2 M_{2} \omega_{n}}+\frac{k_{34}(\tau) f\left(\delta_{2}\right)}{M_{2} \omega_{n}^{2}}-\frac{F_{L}}{b_{c} m_{4} \omega_{n}^{2}} \\
-2 \frac{c_{\alpha_{2}} \dot{\delta_{4}}}{2 m_{3} R_{b 3}^{2} \omega_{n}}-\frac{k_{\alpha 2}}{\delta}{ }_{4} m_{3} R_{b 3}^{2} \omega_{n}^{2}=-\ddot{e}_{34}(\tau) \\
\ddot{\delta}_{3}-2 \frac{c_{12} \dot{\delta_{1}}}{2 m_{2} \omega_{n}}-\frac{k_{12}(\tau) f\left(\delta_{1}\right)}{m_{2} \omega_{n}^{2}}-\frac{F_{c 1}}{b_{c} m_{c 1} \omega_{n}^{2}} \\
+2 \frac{c_{\alpha_{1}} \dot{\delta_{3}}}{2 M_{2 . c 1} R_{b 2}^{2} \omega_{n}}+\frac{k_{\alpha 1}}{\delta}{ }_{3} M_{2 . c 1} R_{b 2}^{2} \omega_{n}^{2}=0 \\
\ddot{\delta}_{4}-2 \frac{c_{34} \dot{\delta_{4}}}{2 m_{3} \omega_{n}}-\frac{k_{34}(\tau) f\left(\delta_{2}\right)}{m_{3} \omega_{n}^{2}}-\frac{F_{c 2}}{b_{c} m_{c 2} \omega_{n}^{2}} \\
+2 \frac{c_{\alpha_{2}} \dot{\delta}_{4}}{2 M_{c 2.3} R_{b 3}^{2} \omega_{n}}-\frac{k_{\alpha 2}}{\delta} M_{42.3} R_{b 3}^{2} \omega_{n}^{2}=0 \\
\ddot{\delta}_{c}-2 \frac{c_{\alpha_{1}} \dot{\delta_{3}}}{2 m_{c 1} R_{b 2}^{2} \omega_{n}}-\frac{k_{\alpha_{1}}\left(\delta_{3}\right)}{m_{c 1} R_{b 2}^{2} \omega_{n}^{2}}-2 \frac{c_{\alpha_{2}} \dot{\delta_{4}}}{2 m_{c 2} R_{b 3}^{2} \omega_{n}} \\
-\frac{k_{\alpha 2}}{\delta} m_{c 2} R_{b 3}^{2} \omega_{n}^{2}+\frac{F_{c 1}}{b_{c} m_{c 1} \omega_{n}^{2}}+\frac{F_{c 2}}{b_{c} m_{c 2} \omega_{n}^{2}}=0
\end{array}\right.
$$

where: $k_{12(34)}(\tau)=k_{01(2)}+\sum_{i=1}^{n} k_{h i} \cos \left(i \omega_{h 1(2)}-\emptyset_{h i}\right)$; $e_{12(34)}(\tau)=\frac{e_{a i}}{b_{c}} \cos \left(\omega_{h i}(\tau)-\emptyset_{h i}\right) ; \omega_{h 1}=\frac{\omega_{1}}{\omega_{n}} ; \omega_{h 2}=$ $\frac{\omega_{2}}{;} \omega_{n}=\sqrt{\frac{K_{01}}{M_{1}}} ; f(\delta)=\left\{\begin{array}{l}\delta_{i}-\bar{b}_{i}, \delta_{i}>\bar{b}_{i} \\ 0,\left|\delta_{i}\right| \leq \bar{b}_{i} \\ \delta_{i}+\bar{b}_{i}, \delta_{i}<-\bar{b}_{i}\end{array} \quad \bar{b}_{i}=\frac{b_{i}}{b_{c}}(i=\right.$ $1,2)$; The one-way clutch model can be transformed into the following form:

$$
\begin{aligned}
& F_{c 1}=\frac{K_{c} b_{c}^{a}}{R_{b 2}^{a+1}} f_{c}\left(\delta_{c}\right)^{a}+\frac{C_{c} b_{c}^{b+c} \omega_{n}^{b}}{R_{b 2}^{b+c+1}} \dot{f}_{c}\left(\delta_{c}\right)^{b} f_{c}\left(\delta_{c}\right)^{c} ; \\
& F_{c 2}=\frac{K_{c} b_{c}^{a}}{R_{b 3}^{a+1}} f_{c}\left(\delta_{c}\right)^{a}+\frac{C_{c} b_{c}^{b+c} \omega_{n}^{b}}{R_{b 3}^{b+c+1}} \dot{f}_{c}\left(\delta_{c}\right)^{b} f_{c}\left(\delta_{c}\right)^{c} .
\end{aligned}
$$

Replacing the transformed one-way clutch model into Eq. (7), the system dimensionless equation is obtained as follows:

$$
\left\{\begin{array}{l}
\ddot{\delta}_{1}+2 \xi_{11} \dot{\delta}_{1}+\kappa_{11} f\left(\delta_{1}\right)-2 \xi_{12} \dot{\delta}_{3}-\kappa_{12} \delta_{3} \\
-P_{D}=\ddot{e}_{12}(\tau) \\
\ddot{\delta}_{2}+2 \xi_{21} \dot{\delta}_{2}+\kappa_{21} f\left(\delta_{2}\right)-2 \xi_{22} \dot{\delta_{4}}-\kappa_{22} \delta_{4} \\
-P_{L}=\ddot{e}_{34}(\tau) \\
\ddot{\delta}_{3}-2 \xi_{31} \dot{\delta_{1}}-\kappa_{31} f\left(\delta_{1}\right)+2 \xi_{32} \dot{\delta_{3}}+\kappa_{32} \delta_{3} \\
-2 \xi_{33} \dot{f}_{c}\left(\delta_{c}\right)^{b} f_{c}\left(\delta_{c}\right)^{c}-\kappa_{33} f_{c}\left(\delta_{c}\right)^{a}=0 \\
\ddot{\delta}_{4}-2 \xi_{41} \dot{\delta_{2}}-\kappa_{41} f\left(\delta_{2}\right)+2 \xi_{42} \dot{\delta_{4}}+\kappa_{42} \delta_{4} \\
-2 \xi_{43} \dot{f}_{c}\left(\delta_{c}\right)^{b} f_{c}\left(\delta_{c}\right)^{c}-\kappa_{43} f_{c}\left(\delta_{c}\right)^{a}=0 \\
\ddot{\delta}_{c}-2 \xi_{51} \dot{\delta_{3}}-\kappa_{51} \delta_{3}-2 \xi_{52} \dot{\delta_{4}}-\kappa_{52} \delta_{4} \\
+2 \xi_{33} \dot{f}_{c}\left(\delta_{c}\right)^{b} f_{c}\left(\delta_{c}\right)^{c}+\kappa_{33} f_{c}\left(\delta_{c}\right)^{a} \\
+2 \xi_{43} \dot{f}_{c}\left(\delta_{c}\right)^{b} f_{c}\left(\delta_{c}\right)^{c}+\kappa_{43} f_{c}\left(\delta_{c}\right)^{a}=0
\end{array}\right.
$$

Where: $\xi_{11}=\frac{c_{12}}{2 M_{1} \omega_{n}} ; \xi_{12}=\frac{c_{\alpha 1}}{2 R_{b 2}^{2} m_{2} \omega_{n}} ; \kappa_{11}=$ $\frac{k_{12}(\tau)}{M_{1} \omega_{n}^{2}} ; \kappa_{12}=\frac{k_{\alpha 1}}{R_{b 2}^{2} m_{2} \omega_{n}^{2}} ; \xi_{21}=\frac{c_{34}}{2 M_{2} \omega_{n}} ; \xi_{22}=$

$$
\begin{aligned}
& \frac{c_{\alpha 2}}{2 R_{b 3}^{2} m_{3} \omega_{n}} ; \kappa_{21}=\frac{k_{34}(\tau)}{M_{21} \omega_{n}^{2}} ; \kappa_{22}=\frac{k_{\alpha 2}}{R_{b 3}^{2} m_{3} \omega_{n}^{2}} ; \xi_{31}= \\
& \frac{c_{12}}{2 m_{2} \omega_{n}} ; \xi_{32}=\frac{c_{\alpha 1}}{2 R_{b 2}^{2} M_{2 . c 1} \omega_{n}} ; \xi_{33}=\frac{c_{c} b_{c}^{b+c+1} \omega_{n}^{b-2}}{2 R_{b 2}^{b+c+1} m_{c 1}} ; \kappa_{31}= \\
& \frac{k_{12}(\tau)}{m_{2} \omega_{n}^{2}} ; \kappa_{32}=\frac{k_{\alpha 1}}{R_{b 2}^{2} M_{2 . c 1} \omega_{n}^{2}} ; \kappa_{33}=\frac{k_{c} b_{c}^{a-1}}{R_{b 2}^{a+1} m_{c 1} \omega_{n}^{2}} ; \xi_{41}= \\
& \frac{c_{34}}{2 m_{3} \omega_{n}} ; \xi_{42}=\frac{c_{\alpha 2}}{2 R_{b 3}^{2} M_{c 2.3} \omega_{n}} ; \xi_{43}=\frac{c_{c} b_{c}^{b+c+1} \omega_{n}^{b-2}}{2 R_{b 3}^{b+c+1} m_{c 2}} ; \kappa_{41}= \\
& \frac{k_{34}(\tau)}{m_{3} \omega_{n}^{2}} ; \kappa_{42}=\frac{k_{\alpha 2}}{R_{b 3}^{2} M_{c 2.3} \omega_{n}^{2}} ; \kappa_{43}=\frac{k_{c} b_{c}^{a-1}}{R_{b 3}^{a+1} m_{c 2} \omega_{n}^{2}} ; \xi_{51}= \\
& \frac{c_{\alpha 1}}{2 R_{b 2}^{2} m_{c 1} \omega_{n}} ; \xi_{52}=\frac{c_{\alpha 2}}{2 R_{b 3}^{2} m_{c 2} \omega_{n}} ; \kappa_{51}=\frac{k_{\alpha 1}}{R_{b 2}^{2} m_{c 1} \omega_{n}^{2}} ; \kappa_{52}= \\
& \frac{k_{\alpha 2}}{R_{b 3}^{2} m_{c 2} \omega_{n}^{2}} ; P_{D}=\frac{F_{D}}{b_{c} m_{1} \omega_{n}^{2}} ; P_{L}=\frac{F_{L}}{b_{c} m_{4} \omega_{n}^{2}} . \text { By processing } \\
& P_{n}(9), t_{n}
\end{aligned}
$$

Eq. (9), the system dimensionless equation can be transformed into the following equation:

$$
\left\{\begin{array}{l}
\ddot{\delta}_{1}=-\ddot{e}_{12}(\tau)-2 \xi_{11} \dot{\delta_{1}}-\kappa_{11} f\left(\delta_{1}\right) \\
+2 \xi_{12} \dot{\delta_{3}}+\kappa_{12} \delta_{3}+P_{D} \\
\ddot{\delta}_{2}=-\ddot{e}_{34}(\tau)-2 \xi_{21} \dot{\delta_{1}}-\kappa_{21} f\left(\delta_{2}\right) \\
+2 \xi_{22} \dot{\delta_{4}}+\kappa_{22} \delta_{4}+P_{L} \\
\ddot{\delta}_{3}=2 \xi_{31} \dot{\delta_{1}}+\kappa_{31} f\left(\delta_{1}\right)-2 \xi_{32} \dot{\delta_{3}}-\kappa_{32} \delta_{3} \\
+2 \xi_{33} \dot{f}_{c}\left(\delta_{c}\right)^{b} f_{c}\left(\delta_{c}\right)^{c}-\kappa_{33} f_{c}\left(\delta_{c}\right)^{a} \\
\ddot{\delta}_{4}=2 \xi_{41} \dot{\delta_{2}}+\kappa_{41} f\left(\delta_{2}\right)-2 \xi_{42} \dot{\delta_{4}}-\kappa_{42} \delta_{4} \\
+2 \xi_{43} \dot{f}_{c}\left(\delta_{c}\right)^{b} f_{c}\left(\delta_{c}\right)^{c}+\kappa_{43} f_{c}\left(\delta_{c}\right)^{a} \\
\ddot{\delta}_{c}=2 \xi_{51} \dot{\delta_{3}}+\kappa_{51} \delta_{3}+2 \xi_{52} \dot{\delta_{4}}-\kappa_{52} \delta_{4} \\
-2 \xi_{33} \dot{f}_{c}\left(\delta_{c}\right)^{b} f_{c}\left(\delta_{c}\right)^{c}-\kappa_{33} f_{c}\left(\delta_{c}\right)^{a} \\
-2 \xi_{43} \dot{f}_{c}\left(\delta_{c}\right)^{b} f_{c}\left(\delta_{c}\right)^{c}-\kappa_{43} f_{c}\left(\delta_{c}\right)^{a}
\end{array}\right.
$$

\section{PARAMETRIC STUDY}

The composition of the gear is 20CrMnTi. The composition of the clutch wedge and inner/outer ring are GCr15 and $18 \mathrm{CrNi} 4 \mathrm{~A}$. According to the calculation formula and the material parameters of the selected parts. Table 1 presents the main parameters of the system. The module of the gear module and the torsional stiffness of the clutch are used as the control parameters to study the influence of the gear module and torsional stiffness on the dynamic transmission error and meshing force of the system. Moreover, the effect of structural parameters of the gear on the dynamic slip angle of the one-way clutch is also studied.

The vibration characteristics of the system are described by the (DTE) and the ODTE (Oscillating DTE). According to the study of, ${ }^{25}$ the DTE is given in the form of root mean square and the following equation is obtained:

$$
\begin{gathered}
R M S \text { of } D T E=\sqrt{\frac{\sum_{i=1}^{N}\left(D T E_{i}\right)^{2}}{N}} ; \\
O D T E=\sqrt{\frac{\sum_{i=1}^{N}\left(D T E_{i+1}-D T E_{i}\right)^{2}}{N}} .
\end{gathered}
$$

In order to study the maximum meshing force that the gear can bear during the working process as the excitation fre- 
quency changes, the dynamic load factor $K_{v}{ }^{25}$ is used as follows:

$$
K_{v}=\frac{F_{d \max }}{F_{n}}=\frac{\max _{i=1, n}\left(k_{i} x_{i}+c_{i} \dot{x}\right)}{F_{n}} ;
$$

where: $k_{i} x_{i}$ and $c_{i} \dot{x}_{i}$ represent the stiffness and damping terms of the load, respectively. Moreover, $F_{n}$ is the torque transmitted by the gear pair (or clutch).

\subsection{The Influence of Different Gear Modules on the Gear Transmission Error}

In this section, the effect of different gear modules of the gear pair 1 and 2 within the range of 2, 2.5, 3, 4, 5, 6, and 8 on gear transmission error is investigated. It should be indicated that other parameters are kept as standard values.

Figure 3 shows that during acceleration and deceleration, the dynamic error response curve of the gear pair 1 shows non-linear characteristics such as jumps. During the acceleration process, in the low-frequency band, when the module is smaller the dynamic transmission error of the gear, pair 1 will fluctuate nonlinearly as the excitation frequency increases. When the module is larger, the change is not obvious and the excitation frequency is within $0.59 \leq \omega_{h} \leq 0.94$. It is worth noting that the dynamic transmission error of pair 1 shows a linear increasing trend. Moreover, under the same excitation frequency, the smaller the module, the greater the dynamic transmission error. When the excitation frequency is within $0.98 \leq \omega_{h} \leq 1.16,1.47 \leq \omega_{h} \leq 2.5$, the gear pair 1 is dynamic and the transmission error is not affected by the module. During the deceleration process, the dynamic error response curve in the low-frequency range shows a non-linear change as the excitation frequency increases. It should be noted that the smaller the module, the more obvious the change, and it is not affected by the module in the high-frequency band. Compared with the acceleration process, the biggest difference is that the amplitude of the dynamic transmission error of the system is significantly smaller. In the low-frequency band, the dynamic transmission error of the system during the deceleration process under the same excitation frequency is larger, and the high-frequency range is consistent with the acceleration process. Since the dynamic behavior of the system during acceleration and deceleration is relatively similar, the present study mainly focused on the acceleration process.

Figure 4 shows that in the low-frequency band when the excitation frequency is within $0.1 \leq \omega_{h} \leq 0.59$, the gear pair 1 shows an obvious jumping phenomenon. When the module is smaller, the phenomenon is more obvious. When the excitation frequency is within $0.59 \leq \omega_{h} \leq 0.94$, and as the excitation frequency increases, the jumping phenomenon of the gear pair 1 shows an increasing trend. Moreover, under the same excitation frequency, when the module is smaller, the vibration is greater. In the high-frequency band, the module has little effect on the vibration phenomenon of the gear pair 1.

Figure 5 shows that during acceleration, the gear dynamic load decreases monotonously when $0.1 \leq \omega_{h} \leq 0.11$. Increasing the module increases the gear dynamic load when $0.59 \leq \omega_{h} \leq 0.98$ and the excitation frequency corresponding to the peak dynamic load will be higher. Furthermore, in

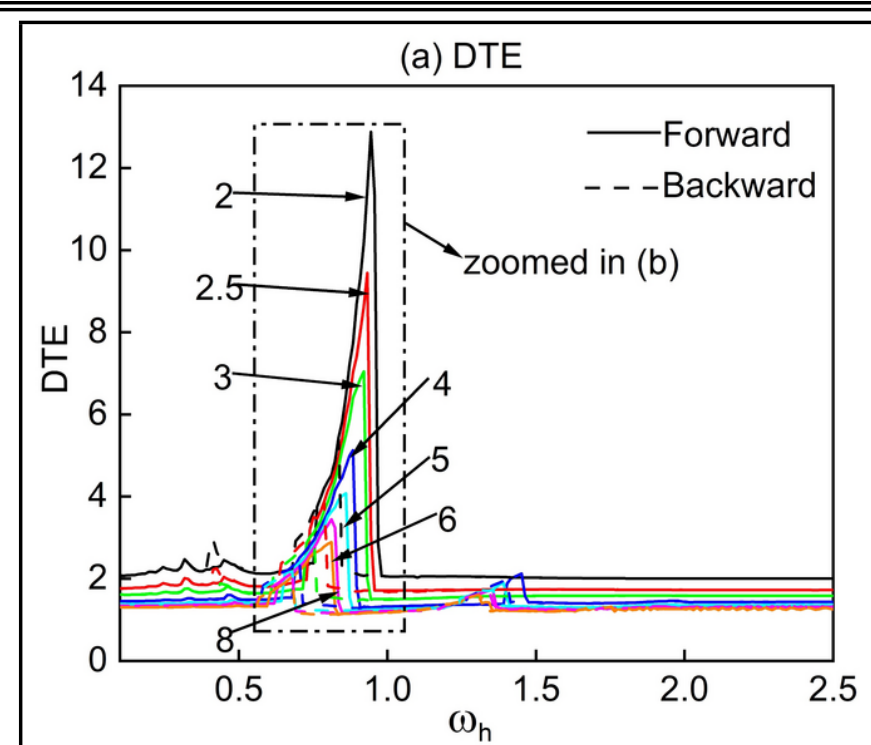

(b) zoomed area of (a)

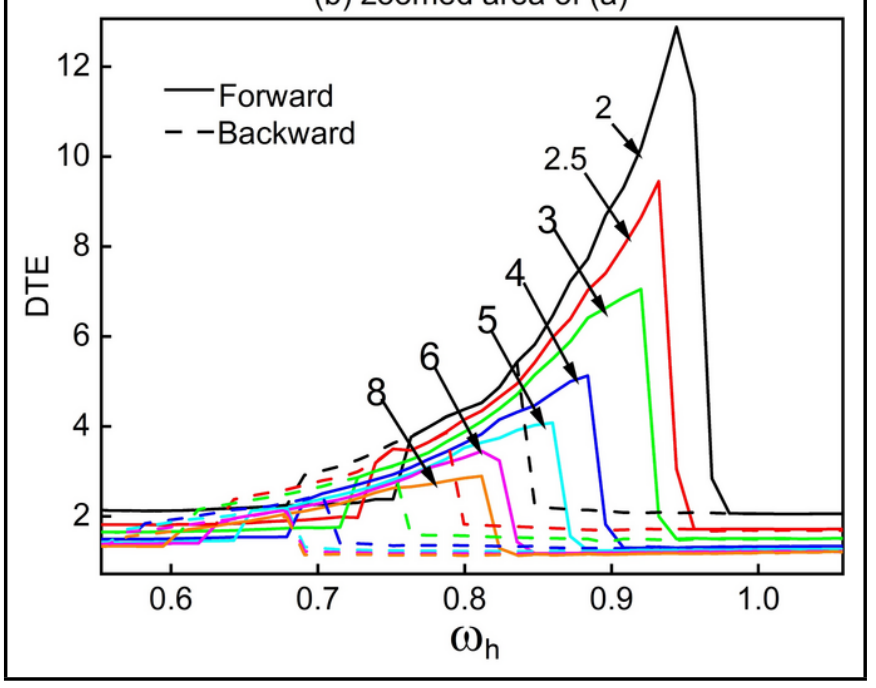

Figure 3. Root mean square value of the dynamic transmission error of gear pair 1 with different gear module:(a) DTE and (b) zoomed area of (a).

the excitation frequency range of $0.98 \leq \omega_{h} \leq 2.03$, as the module increases, the gear pair 1 shows an obvious non-linear jumping phenomenon. As the module increases, the dynamic load increases too. When $2.03 \leq \omega_{h} \leq 2.5$, the module has little effect on the dynamic load of the gear.

Figure 6 shows that the dynamic transmission error response curve of the gear pair 2 which has obvious non-linear characteristics such as jumps. During the acceleration process, when the excitation frequency is within $0.32 \leq \omega_{h} \leq 1.05$, the dynamic transmission error of the gear pair 2 presents a fluctuating nonlinear change as the excitation frequency increases and there is an obvious jump phenomenon. However, the influence of the module on its peak value is not obvious. In the midto-high frequency band, the larger the module, the smaller the dynamic transmission error of the gear pair 2. Moreover, the fl changes with an increase in the excitation frequency which results in the greater the dynamic transmission error of gear pair 2.

Figure 7 shows that in the low-frequency band, an obvious jump phenomenon is observed when the excitation frequency is $0.32 \leq \omega_{h} \leq 1.01$. In the high-frequency band, as the excitation frequency increases, the curve change trend is the 


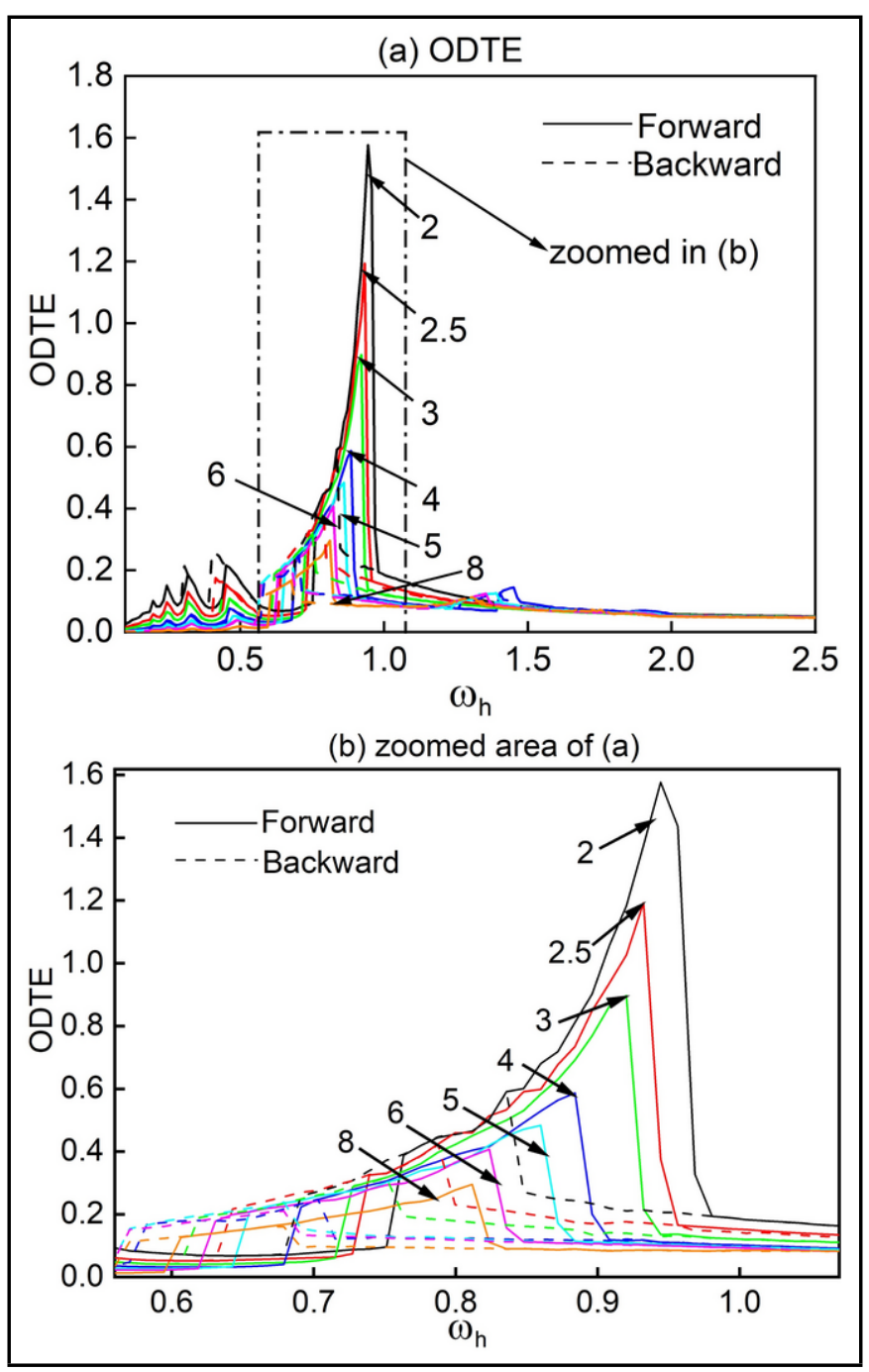

Figure 4. Root mean square error value of dynamic transmission error of gear pair 1 with different gear module:(a) ODTE and (b) zoomed area of (a).

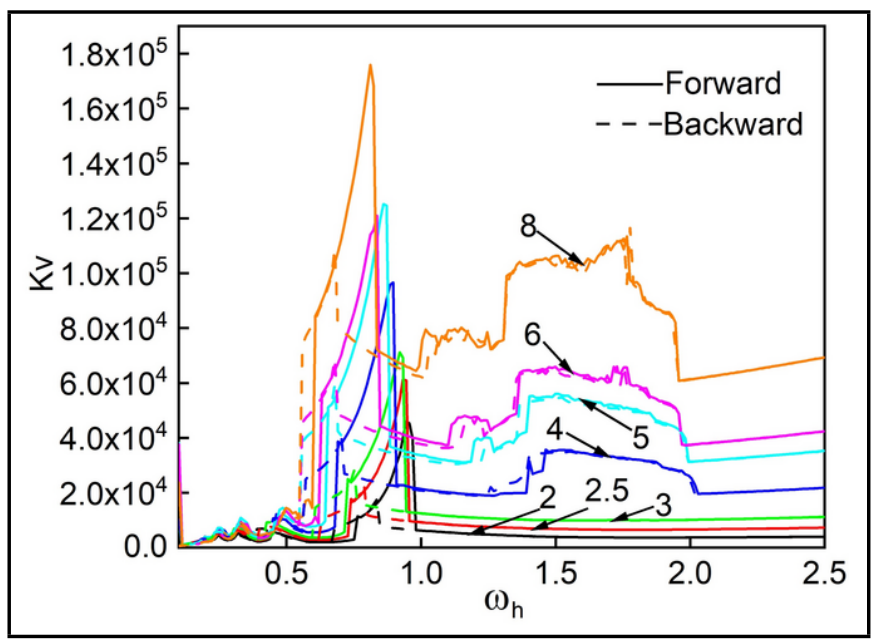

Figure 5. Dynamic load coefficient of gear pair 1 with different gear module.

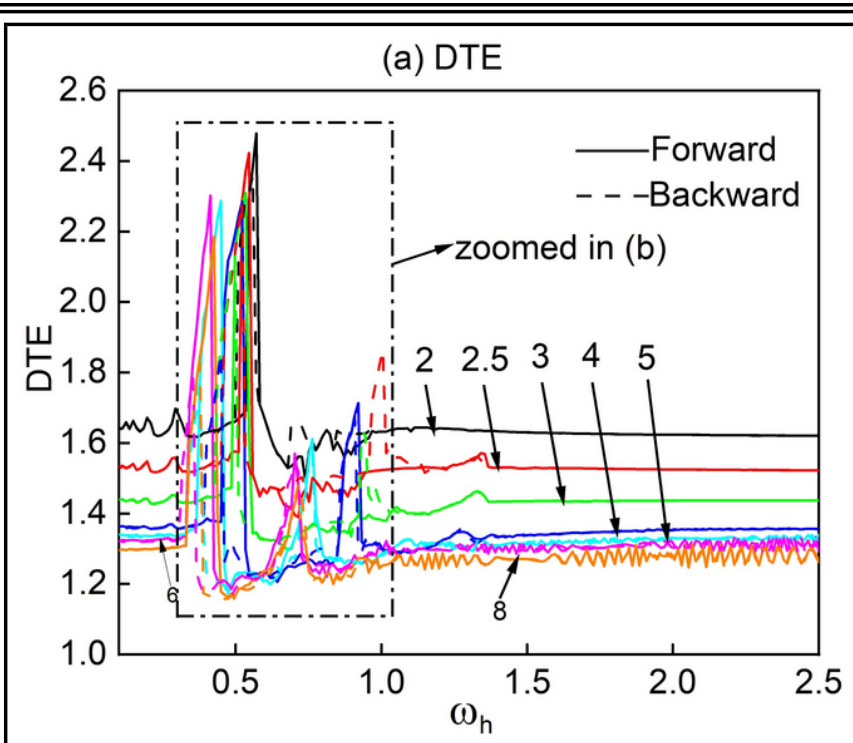

(b) zoomed area of (a)

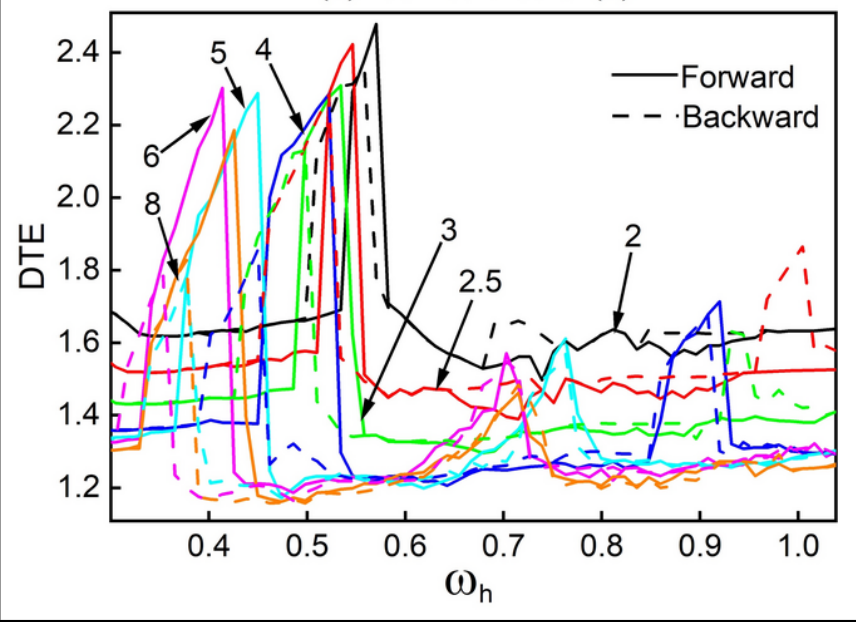

Figure 6. Root mean square value of dynamic transmission error of gear pair 2 with different gear module:(a) DTE and (b) zoomed area of (a).

same. The influence of the module on the variation of ODTE can be ignored.

Figure 8 illustrates that in the low-frequency range of $0.1 \leq$ $\omega_{h} \leq 0.11$, the dynamic load presents a monotonous decreasing trend. Within the range of $0.31 \leq \omega_{h} \leq 0.44$ and of $0.7 \leq \omega_{h} \leq 1.08$, when the module increases, the dynamic load becomes unstable and the maximum dynamic load increases. Within the range of $1.08 \leq \omega_{h} \leq 2.5$, if the module increases, the dynamic load increases.

\subsection{The Influence of Different Gear Modules on the Input and Output Difference of the One-Way Clutch}

The dynamic characteristics of the one-way clutch are usually evaluated by the dynamic windup angle. When the oneway clutch works normally, the outer ring will rotate at a certain angle more than the inner ring assembly to achieve a synchronous movement. This angle difference is the windup angle. The dynamic windup angle refers to the instantaneous angle difference between the outer ring and the inner ring assembly when the clutch is engaged.

Figure 9 illustrates that during the acceleration process, 


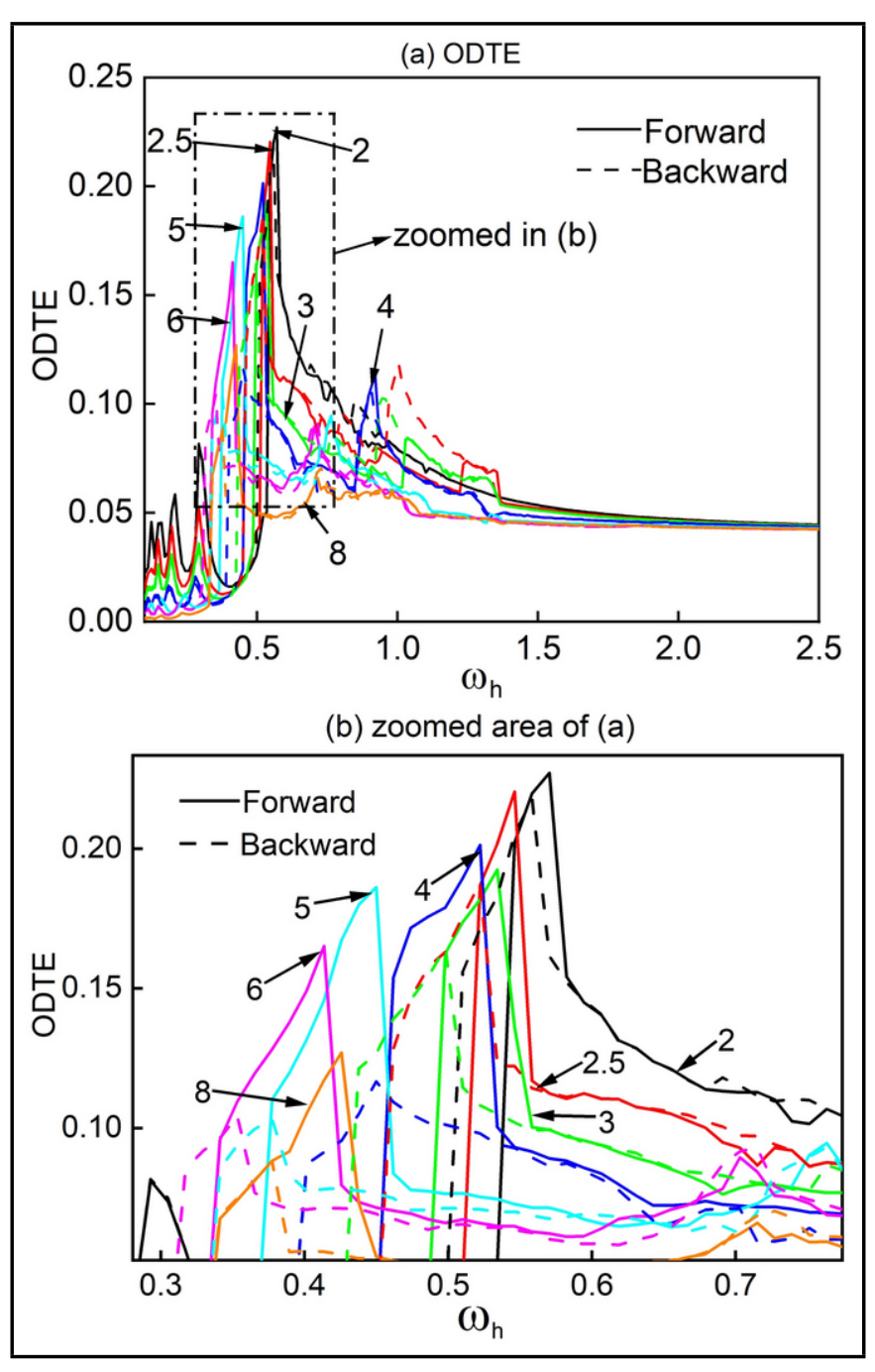

Figure 7. Root mean square error value of dynamic transmission error of gear pair 2 with different gear module:(a) ODTE and (b) zoomed area of (a).

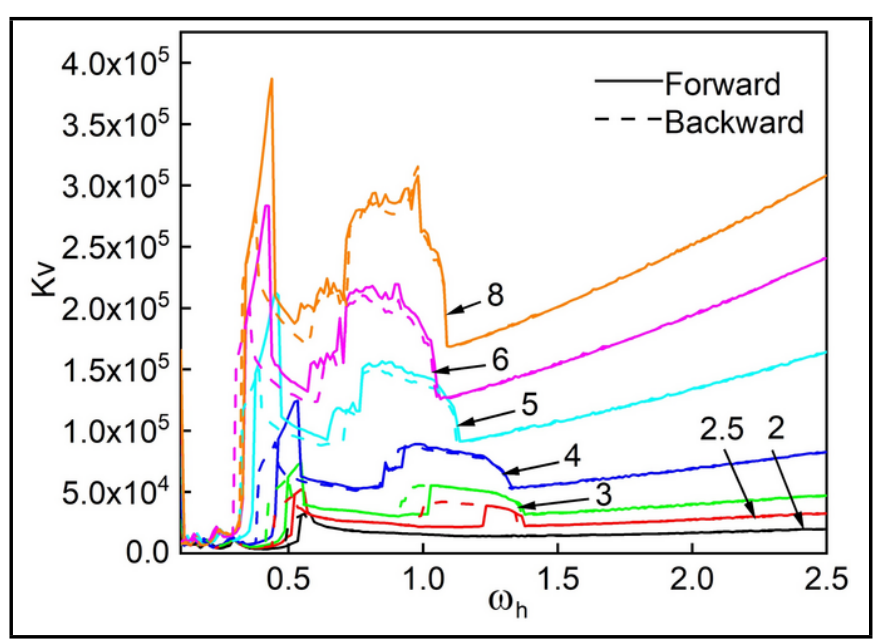

Figure 8. Dynamic load coefficient of gear pair 2 with different gear module.

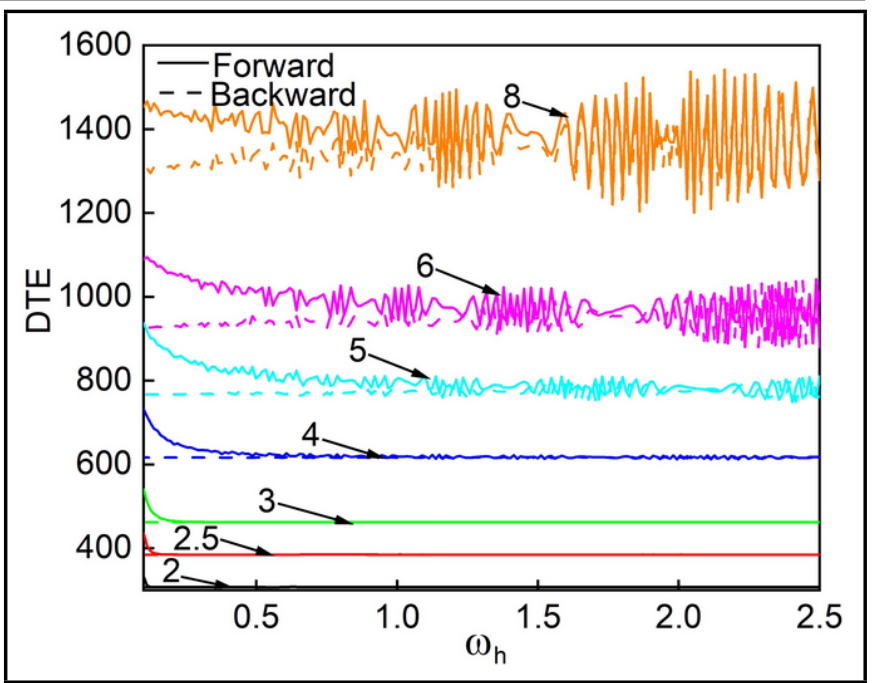

Figure 9. Root mean square value of the dynamic windup angle of the oneway clutch with different gear module.

when the gear module is small ( $m=2,2.5,3$, and 4 in this study), the excitation frequency has little effect on the dynamic windup angle. Within the range of $0.1 \leq \omega_{h} \leq 0.48$, the dynamic windup angle presents a monotonous decreasing trend and maintains a constant value in the forward. The gear module has a greater influence on the dynamic windup angle. If the gear module increases, the dynamic windup angle also increases. When the gear module is larger $(m=5,6$, and 8 in this study), as the excitation frequency increases, the dynamic windup angle fluctuates. Moreover, the greater the gear module, the greater the fluctuation. During the deceleration process, the excitation frequency has little effect on the dynamic windup angle and maintains a constant value. It should be noted that the gear module has the same effect on the clutch dynamic windup angle as the acceleration process.

Figure 10 shows that during the acceleration process, when the excitation frequency is within the range of $0.1 \leq \omega_{h} \leq$ 0.44 , the dynamic windup angle changes in a monotonous decreasing trend. The smaller the module, the greater the magnitude of the change. Within the range of $0.45 \leq \omega_{h} \leq 1.41$, when $m=5,6$ the clutch dynamic windup angle shows obvious jumps as the excitation frequency increases. When the excitation frequency is in the range of $1.82 \leq \omega_{h}$, the clutch dynamic windup angle has little influence on the gear module keeping and maintaining stability.

\subsection{The Influence of Different Clutch Torsional Stiffnesses on the Gear Dynamic Error}

The gear module of 5 and other parameters of the system are consistent with Table 1 . When the stiffness of the one-way clutch changes within the range of 0.1 to 10 multiples, it can cover the selection range of the rigidity of the commonly used materials of the one-way clutch.

Figure 11 shows that during the acceleration process, the dynamic transmission error of the gear pair 1 has an obvious jump phenomenon. In the frequency band of $0.1 \leq \omega_{h} \leq 0.28$, the clutch torsional stiffness does not have an obvious influence on dynamic transmission error. Within the range of 


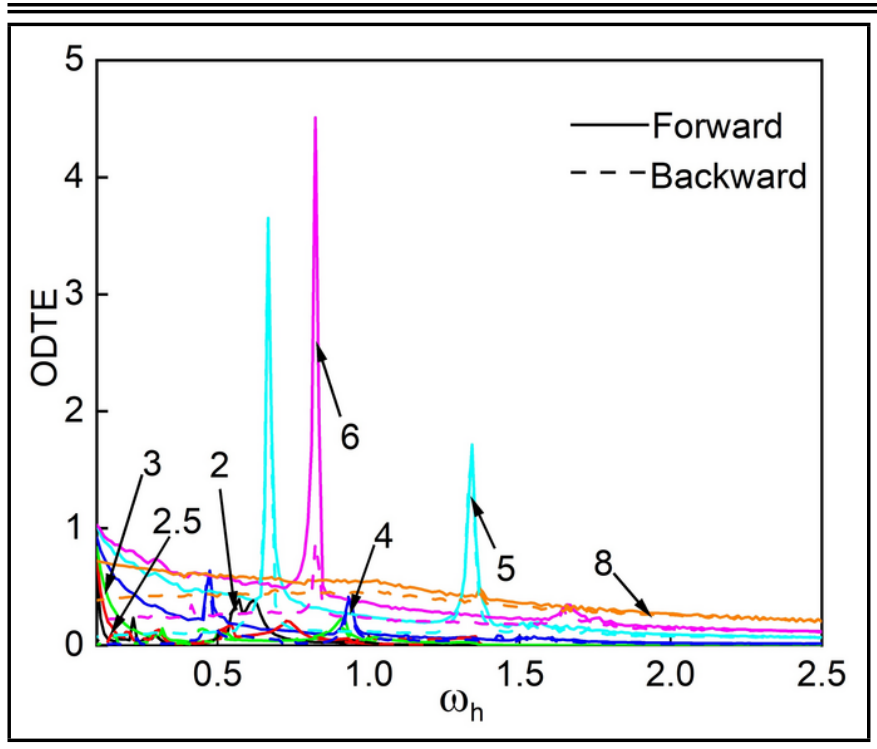

Figure 10. Root mean square error value of the dynamic windup angle of the one-way clutch with different gear module.

Table 1. System assembly attributes.

\begin{tabular}{||c|c|c|}
\hline Parameter name & Parameter code & Parameter value \\
\hline Gear & $z_{1}$ & \\
\hline \multirow{3}{*}{ Gear teeth } & $z_{2}$ & 65 \\
\cline { 2 - 3 } & $z_{3}$ & 80 \\
\cline { 2 - 3 } & $z_{4}$ & 95 \\
\hline Pressure angle & $\alpha$ & 110 \\
\hline Contact ratio & $\varepsilon_{1}$ & 1.8113 \\
\cline { 2 - 3 } & $\varepsilon_{1}$ & 1.8547 \\
\hline Meshing damping & $\zeta_{1}, \zeta_{2}$ & 0.05 \\
\hline One-way clutch & \multicolumn{2}{|c}{} \\
\hline $\begin{array}{c}\text { Damping ratio of active } \\
\text { end and driven end }\end{array}$ & $z_{c}$ & 0.026 \\
\hline
\end{tabular}

$0.29 \leq \omega_{h} \leq 0.54$, the dynamic transmission error response curve shows small fluctuations. The excitation frequency is within the range of $0.55 \leq \omega_{h} \leq 0.88$ and $1.07 \leq \omega_{h} \leq 1.46$, when the increase of the excitation frequency that the dynamic transmission error of gear pair 1 changes significantly and in the same excitation frequency if the greater the torsional stiffness results in the smaller the peak value of the dynamic transmission error. In the high-frequency band when the torsional stiffness of the clutch of 5 and 10 multiples, the dynamic transmission error of gear pair 1 appears to fluctuate nonlinearly with the increase of excitation frequency.

Figure 12 shows that in the low-frequency band, the torsional stiffness has little effect on the gear pair 1 . When the excitation frequency is within the range of $0.58 \leq \omega_{h} \leq 0.88$, an obvious jump phenomenon is observed. The clutch torsional stiffness affects the gear pair noise and vibration noticeably. Moreover, the increment of the torsional stiffness of the clutch can suppress the vibration of the gear pair and reduce the noise emitted by the gear pair during operation. At the frequency jump ( $\left.\omega_{h}=0.85\right)$, the greater the torsional stiffness, the smaller the peak.

Figure 13 shows that in the low-frequency range, reducing the torsional stiffness of the one-way clutch can reduce the maximum dynamic load of the gear pair 1. Moreover, at the frequency jump $\omega_{h}=0.87$, as the torsional stiffness increases, (a) DTE

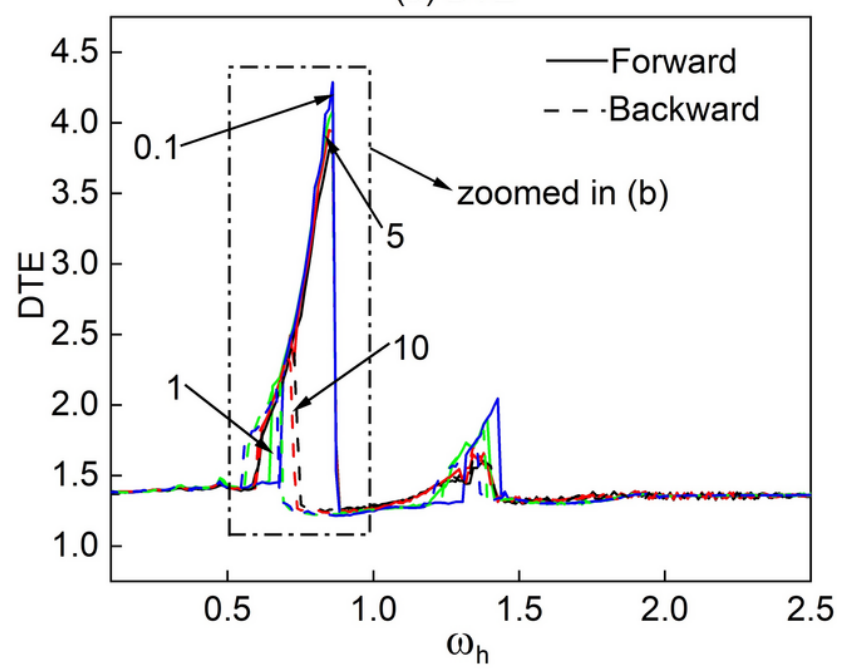

(b) zoomed area of (a)

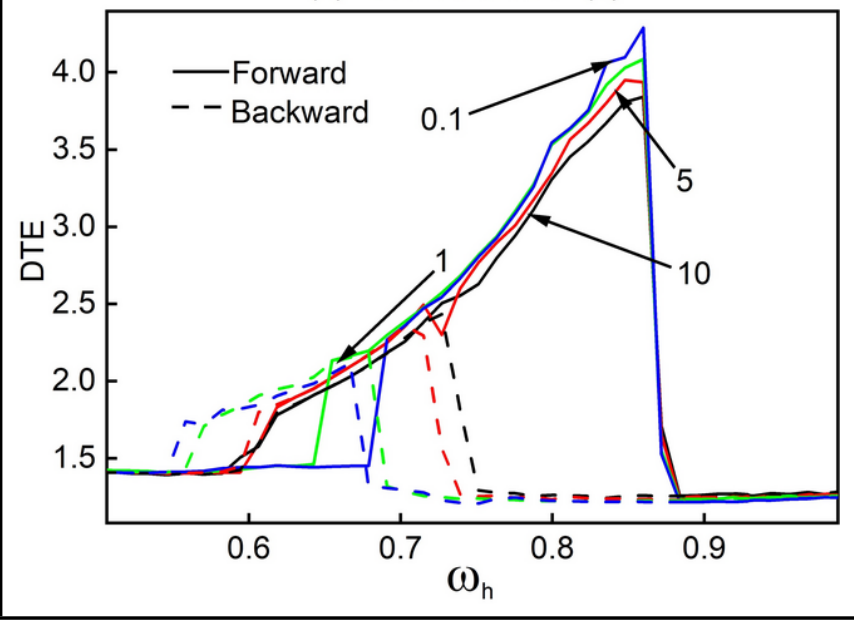

Figure 11. Root mean square value of the dynamic transmission error of gear pair 1 with different torsional stiffness of the one-way clutch :(a) DTE and (b) zoomed area of (a).

the dynamic load increases. At the excitation frequency within the range of $0.99 \leq \omega_{h} \leq 2$, the system shows an obvious chaotic response and the greater the torsional stiffness, the greater the dynamic load. Furthermore, in the high-frequency range, the maximum dynamic load is not affected by the torsional stiffness of the one-way clutch.

Figure 14 shows that in the frequency band $\omega_{h}=0.44$ and $0.65 \leq \omega_{h} \leq 0.80$, an obvious jumping phenomenon is observed. In the high-frequency band, when the torsional stiffness is 0.1 multiple, the dynamic transmission error fluctuation amplitude of the gear pair 2 is significantly reduced.

Figure 15 shows that in the low-frequency band, especially at $\omega_{h}=0.44$, an obvious jumping phenomenon is observed. When the torsional stiffness is 1 , the peak value is the highest. Moreover, when the torsional stiffness value is $0.1,5$, and 10 multiples, the peak value can be effectively suppressed. In the frequency range of $0.61 \leq \omega_{h} \leq 1.5$, the vibration and noise of the gear pair 2 during operation will show unstable fluctuations and nonlinear changes as the excitation frequency increases. When the torsional stiffness value is 5 and 10 multiples, the magnitude and peak value of small fluctuations can be effectively reduced. 


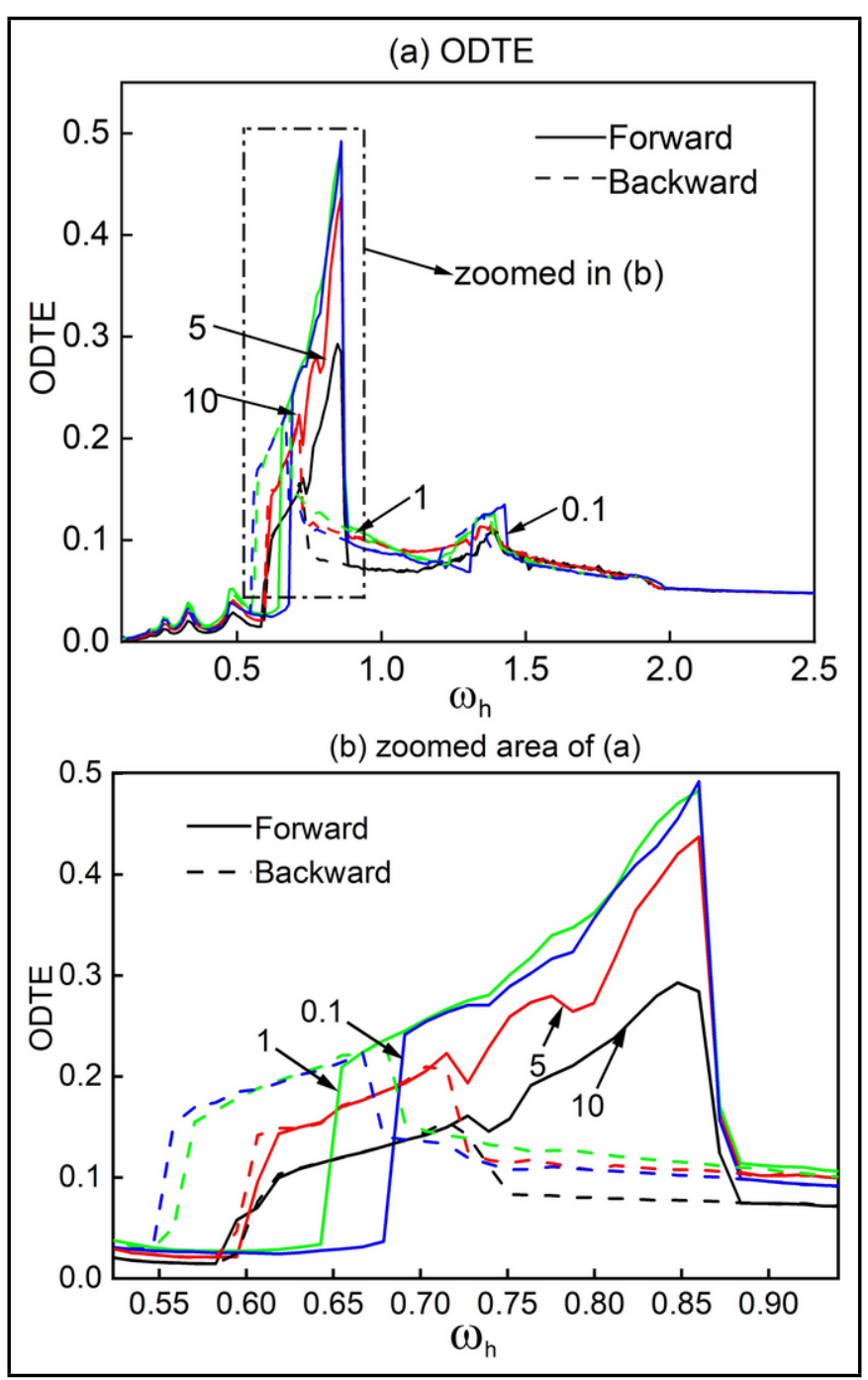

Figure 12. Root mean square error value of the dynamic transmission error of gear pair 1 with different torsional stiffness of the one-way clutch :(a) ODTE and (b) zoomed area of (a).

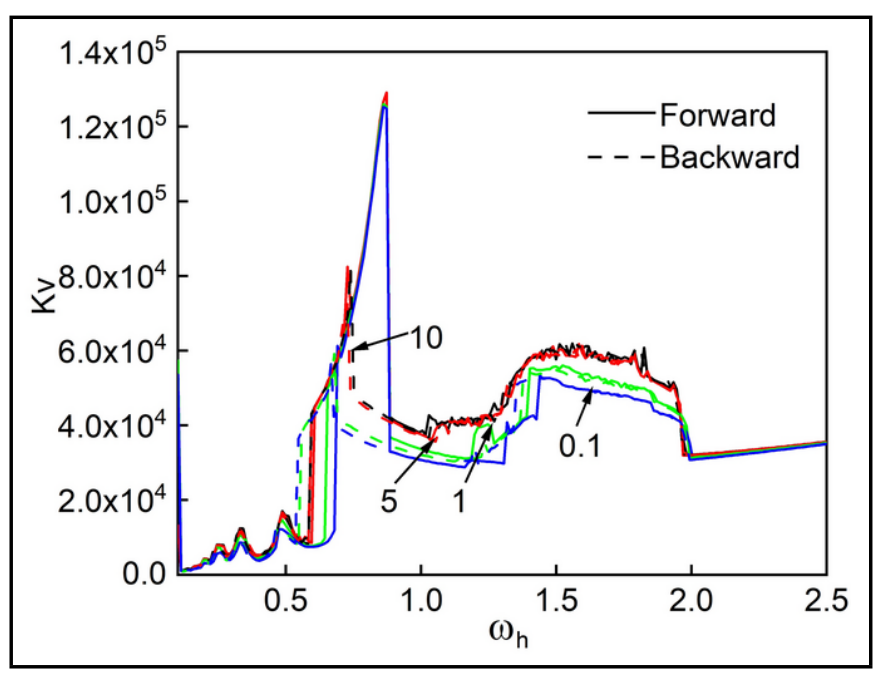

Figure 13. Dynamic load coefficient of gear pair 1 with different torsional stiffness of the one-way clutch. (a) DTE

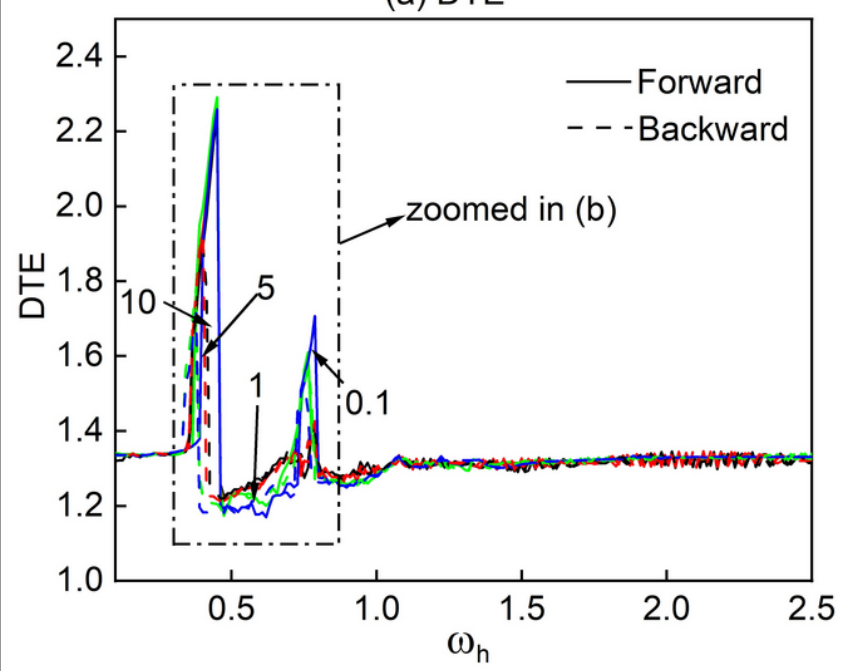

(b) zoomed area of (a)

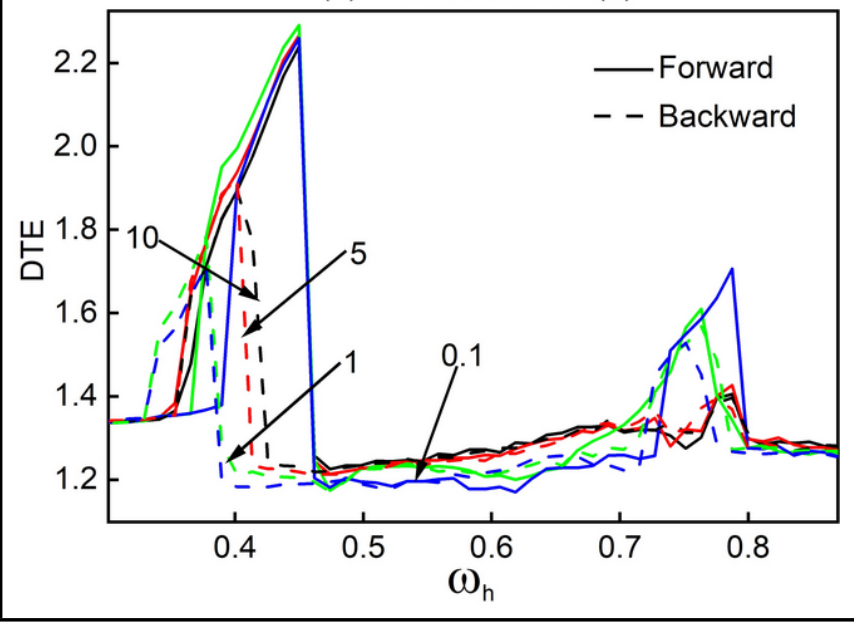

Figure 14. Root mean square value of dynamic transmission error of gear pair 2 with different torsional stiffness of the one-way clutch :(a) DTE and (b) zoomed area of (a).

Figure 16 shows that in the low-frequency range, reducing the torsional stiffness of the one-way clutch can reduce the maximum dynamic load of the gear pair 2. At the frequency jump $\left(\omega_{h}=0.45\right)$, the maximum dynamic load can be controlled by reducing the torsional stiffness. In the frequency range of $0.72 \leq \omega_{h} \leq 1.13$, the system shows an obvious chaotic response. Moreover, the higher the torsional stiffness, the greater the dynamic load. In the high-frequency range, the maximum dynamic load is not affected by the torsional stiffness of the one-way clutch.

\section{CONCLUSIONS}

In the present study, a nonlinear dynamic model of a twostage gear transmission system with a one-way clutch was established. Moreover, the influence of structural parameters such as gear module and clutch torsional stiffness on the dynamic performance of the system was investigated. The following conclusions were drawn from this study:

1. In order to make the gear pair of the system have a relatively good dynamic performance during operation and at the same time reduce the dynamic transmission error 


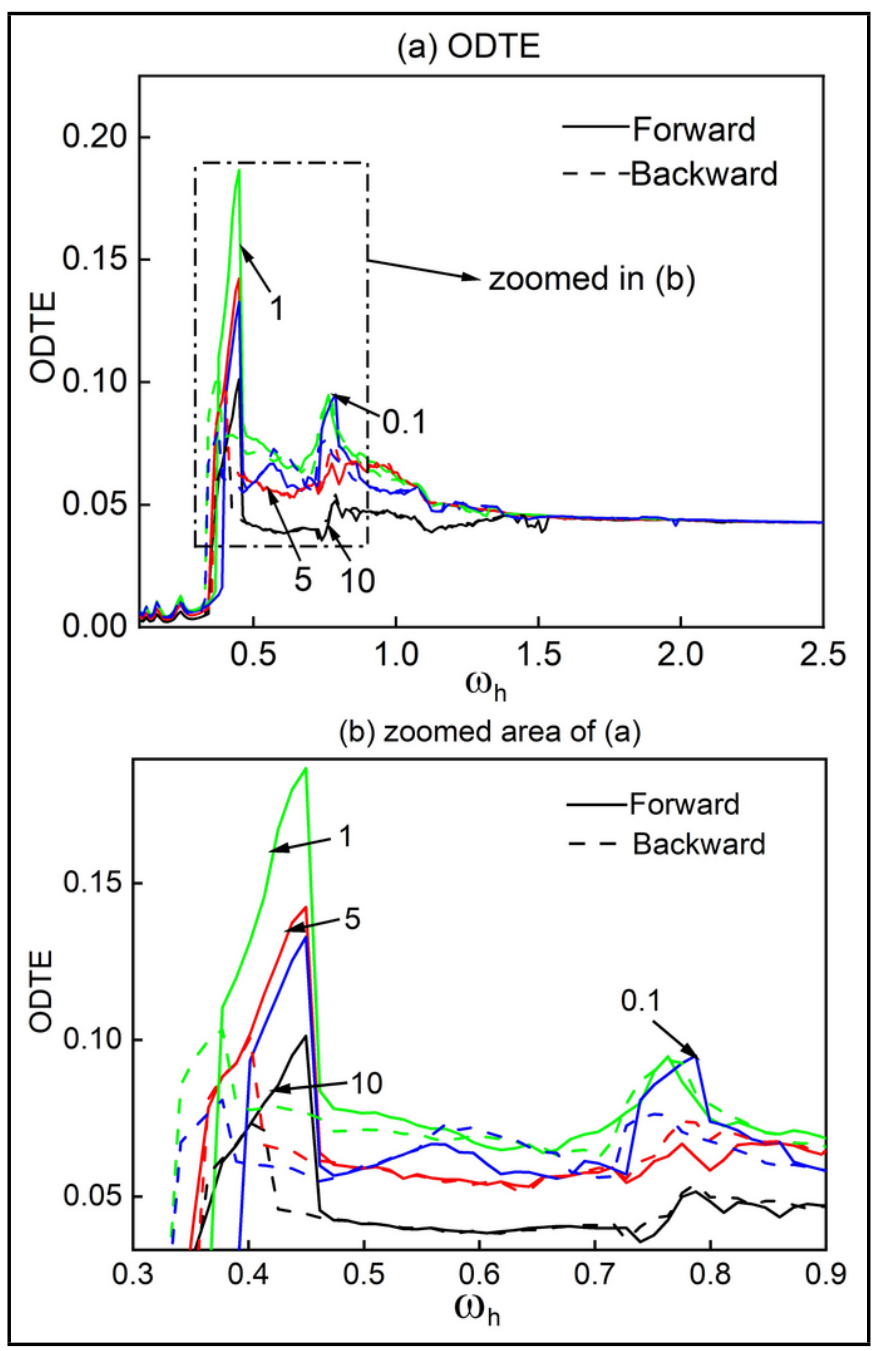

Figure 15. Root mean square value of dynamic transmission error of gear pair 2 with different torsional stiffness of the one-way clutch :(a) DTE and (b) zoomed area of (a). and dynamic load of the gear pair, it is more appropriate to select a larger value for the gear module in the frequency band of $\omega_{h} \leq 1$. Within the frequency band of $1 \leq \omega_{h} \leq 2$, selecting a smaller gear module can effectively reduce the dynamic load of the gear pair. On the other hand, in the frequency band of $\omega_{h} \leq 2$, the dynamic transmission error and dynamic load were hardly affected by the gear module.

2. In order to make the one-way clutch have a good dynamic performance during the system operation and reduce the dynamic windup angle of the one-way clutch, a smaller value for the gear module should be selected.

3. It was found that in the low and middle-frequency bands, a larger torsional stiffness of the one-way clutch can suppress the vibration and noise generated by the gear pair during operation. Meanwhile, a smaller dynamic transmission error was obtained. In the high and ultra-high frequency bands, the one-way clutch had little effect on the torsional stiffness. In order to obtain a lower dynamic load, the torsional stiffness of the one-way clutch should not be too high.

\section{ACKNOWLEDGEMENTS}

The authors thank the natural science foundation project in Hunan province (NO.2019JJ50561), outstanding youth project in Hunan province department of education (NO.18B425), national natural science foundation of China (52075552), Hunan high-tech industry science and technology innovation leading program project No.45 (2020GK2033), key scientific research projects of Hunan provincial department of education (20A455), graduate scientific research innovation project in Hunan province (NO.CX20190964), and graduate scientific research innovation project in Shaoyang University (NO.CX2019SY001) for financial support.

\section{REFERENCES}

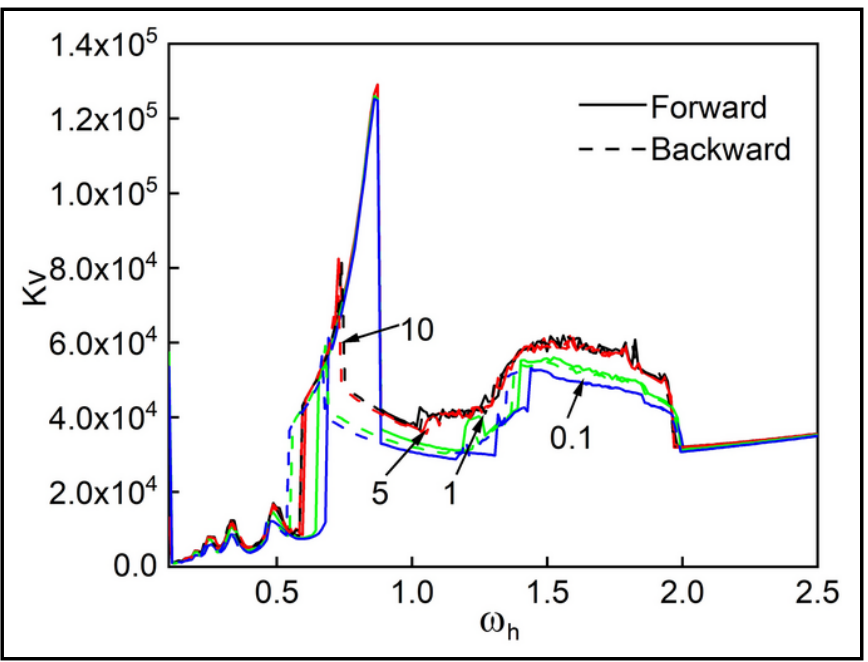

Figure 16. Dynamic load coefficient of gear pair 2 with different torsional stiffness of the one-way clutch.
1 W, A, Tuplin. Gear load capacity, Journal of Applied Mechanics, 30 (3), 479, (1962). https://dx.doi.org/10.1115/1.3636605

2 Sainte-Marie, N., Velex, P., Roulois, G., Caillet, J. A study on the correlation between dynamic transmission error and dynamic tooth loads in spur and helical gears, Journal of Vibration and Acoustics, 139 (1), 011001.1-011001.10, (2017). https://dx.doi.org/10.1115/1.4034631

3 Parker, R, G., Agashe, V., Vijayakar, S, M. Dynamic response of a planetary gear system using a finite element/contact mechanics model, Journal of Mechanical Design, 122 (3), 304-310, (2000). https://dx.doi.org/10.1115/1.1286189

4 Kahraman, A., Singh, R. Non-linear dynamics of a spur gear pair, Journal of Sound and Vibration, 142 (1), 49-75, (1990). https://dx.doi.org/10.1016/0022-460x(90)90582-k 
${ }^{5}$ Chaari, F., Fakhfakh, T., Hbaieb, R., Louati, J., Haddar, $M$. Influence of manufacturing errors on the dynamic behavior of planetary gears, The International Journal of Advanced Manufacturing Technology, 27 (7-8), 738-746, (2005). https://dx.doi.org/10.1007/s00170-004-2240-2

6 Al-Shyyab, A., Kahraman, A. A non-linear dynamic model for planetary gear sets, Proceedings of the Institution of Mechanical Engineers, Part K: Journal of Multi-Body Dynamics, 221 (4), 567-576, (2007). https://dx.doi.org/10.1243/14644193jmbd92

7 Buzzoni, M., D'Elia, G., Mucchi, E., Dalpiaz, G. A vibration-based method for contact pattern assessment in straight bevel gears, Mechanical Systems and Signal Processing, 120, 693-707, (2019). https://dx.doi.org/10.1016/j.ymssp.2018.10.002

${ }^{8}$ Sakaridis, E., Spitas, V., Spitas, C. Non-linear modeling of gear drive dynamics incorporating intermittent tooth contact analysis and tooth eigenvibrations, Mechanism and Machine Theory, 136, 307-333, (2019). https://dx.doi.org/10.1016/j.mechmachtheory.2019.03.012

9 Saxena, A., Parey, A., Chouksey, M. Study of Modal Characteristics of a Geared Rotor System, Procedia Technology, 23, 225-231, (2016). https://dx.doi.org/10.1016/j.protcy.2016.03.021

10 Sun, Z,M., Ji, L,H., Shen, Y, W. Nonlinear dynamics of 2K-H planetary gear train, Journal of Tsinghua University(Science and Technology), 43 (5), 636-639, (2003). https://dx.doi.org/10.16511/j.cnki.qhdxxb.2003.05.015

11 Makoto I, Yukio N, Norikazu K, Hiroshi, H. Development of resin gear balance shaft system for 2AZFE engine, JSAE Review, 23 (1), 27-32, (2002). https://dx.doi.org/10.1016/s0389-4304(01)00164-3

${ }^{12}$ Liu, Z., Liu, Z., Zhao, J., Zhang, G. Study on interactions between tooth backlash and journal bearing clearance nonlinearity in spur gear pair system, Mechanism and Machine Theory, 107, 229-245, (2017). https://dx.doi.org/10.1016/j.mechmachtheory.2016.09.024

13 Wang, G, J., Chen, L., Yu, L., Zou, S,D. Research on the dynamic transmission error of a spur gear pair with eccentricities by finite element method, Mechanism and Machine Theory, 109, 1-13, (2017) https://dx.doi.org/10.1016/j.mechmachtheory.2016.11.006

${ }^{14}$ Liu, X, Z., Yang, Y, H.,Zhang, J. Investigation on coupling effects between surface wear and dynamics in a spur gear system, Tribology International, 101, 383-394, (2016). https://dx.doi.org/10.1016/j.triboint.2016.05.006

$15 \mathrm{~T}, \mathrm{Xu} ., \mathrm{G}, \mathrm{G}$, Lowen. A mathematical model of an over-running sprag clutch, Mechanism and Machine Theory, 29 (1), 11-23, (1994). https://dx.doi.org/10.1016/0094$114 \times(94) 90016-7$
${ }^{16}$ G, G, Lowen., C, Chassapis. The elastic behavior of linkages: An update, Mechanism and Machine Theory, 21 (1), 33-42, (1986). https://dx.doi.org/10.1016/0094$114 \times(86) 90028-5$

${ }^{17}$ D, Centea., H, Rahnejat., M, T, Menday. Non-linear multibody dynamic analysis for the study of clutch torsional vibrations (judder), Applied Mathematical Modelling, 25, 177-192, (2001). https://dx.doi.org/10.1016/s0307$904 \mathrm{x}(00) 00051-2$

18 Yan, H, Z., Yang, B., Hu, K, X., Tan, Y, Q. Working Performance Analysis of Positive-continuous-engagement Type Clutch in Different Friction Conditions, Journal of Mechanical Engineering, 46 (17), 86-92, (2010). https://dx.doi.org/10.3901/jme.2010.17.086

19 Yan, H, Z., Yang, B., Hu, K, X., Tan, Y, Q. Mechanical properties analysis of engaging process of the single arc PCE-type clutch, Journal of China Coal Society, 35 (6), 1034-1038,(2010). https://dx.doi.org/10.13225/j.cnki.jccs.2010.06.030

20 Wu, K., Yan, H, Z., He, M, S., Tan, Y, Q. Dynamics Analysis of Logarithm Curved Surface Sprag PCE Clutch, China Mechanical Engineering, 22 (6), 647-651, (2011). (in Chinese)

21 Yan, H, Z., Wu, K., He, M, S., Tan, Y, Q. Dynamic analysis of archimedes curved surface wedge PCE clutch, Journal of Machine Design, 28 (4), 31-36, (2011). https://dx.doi.org/10.13841/j.cnki.jxsj.2011.04.014

${ }^{22}$ Niranjan Hiremath, D.Mallikarjuna Reddy. An Integrated Tribological and Vibration Signal Behaviour of TiN and AlCrN based PVD Coatings for Roller Bearings, Indian Journal of Science and Technology, 9 (47), 01-08, (2016). https://dx.doi.org/10.17485/ijst/2015/v8i1/107938

${ }^{23}$ Niranjan Hiremath, D.Malikarjuna Reddy. Bearing Fault Detection Using Acoustic Emission Signals Analyzed by Empirical Mode Decomposition, International Journal of Research in Engineering and Technology, 03, 426-431, (2014). https://dx.doi.org/10.15623/ijret.2014.0315084

${ }^{24}$ Cheon, Gill-Jeong. Nonlinear behavior analysis of spur gear pairs with a one-way clutch, Journal of Sound and Vibration, 301 (3-5), 760-776, (2007). https://dx.doi.org/10.1016/j.jsv.2006.10.040

25 Wang, P. Research on Design Methods and Nonlinear Characteristics of the Integrated Controllable Overrunning Clutch System. Doctoral dissertation, Beijing jiaotong University, Beijing, China, (2015).

${ }^{26}$ Reddy M., Kakkar S., Singh P.P., Lath U.S. Modeling and Validation of Ride Characteristics of All-Terrain Vehicle (ATV), Advances in Manufacturing Technology, 18, 547556, (2019). https://dx.doi.org/10.1007/978-981-13-6374$0 \_60$ 